\title{
Controllability of impulsive stochastic functional integrod- ifferential equations driven by Rosenblatt process and Lévy noise
}

\author{
Mahamat Hassan Mahamat Hamit ${ }^{\mathrm{a}}$, Kora Hafiz Bete $^{\mathrm{b}}$, Barka Ibrahim Mahamat ${ }^{\mathrm{a}}$, Mamadou Abdoul Diop ${ }^{\mathrm{a}, \mathrm{c}, *}$ \\ a UFR SAT Département de Mathématiques, Université Gaston Berger de Saint-Louis, B. P234, Saint-Louis, Sénégal. \\ ${ }^{b}$ Institut de Mathématiques et de Sciences Physiques, B.P 613 Porto-Novo, Bénin. \\ ' UMMISCO UMI 209 IRD/UPMC, Bondy, France.
}

\begin{abstract}
In this paper, we develop controllability findings for impulsive neutral stochastic delay partial integrodifferential equations in Hilbert spaces driven by Rosenblatt process and Lévy noise. A novel set of adequate requirements is obtained by utilizing a fixed point method without imposing a stringent compactness constraint on the semigroup. The observed results represent a generalization and continuation of previous findings on this topic. Finally, an example is given to demonstrate how the acquired findings may be used.
\end{abstract}

Keywords: Stochastic functional integrodifferential equations, resolvent operator, rosenblatt process, Lévy noise, controllability. 2020 MSC: 35B35, 39B82, 93E03, 60H15.

(C2022 All rights reserved.

\section{Introduction}

Neutral integrodifferential equations have been the subject of extensive study by several authors over the last many years (see[7, 12, 16, 17, 22, 23, 28, 52] and references therein). Integrodifferential equations of the neutral type are used to represent a wide variety of physical processes that occur in fluid dynamics, electronics, chemical kinetics, and other domains. As a result of their inherent properties, neutral differential equations can be found naturally in the mathematical modeling of a wide range of real phenomena in fields as diverse as mechanics, electronics, control theory, engineering, economics, and statistics. The heat equation developed by Lunardi in [35] and the one used to perform a quantitative assessment of unemployment, wage bills, and income policies in [9] are both good instances of this type of equation. Chukwu [9], provides a number of excellent examples, most of them related to economics. For deterministic neutral functional differential equations, Hale and Mayer [20] were the first to examine them.

Stochastic differential equations have been extensively studied as a mathematical model for describing the dynamical behavior of a real-world phenomenon. The inclusion of environmental disturbances and

\footnotetext{
*Corresponding author

Email addresses: hassan.mahamat-hamit@ugb.edu.sn (Mahamat Hassan Mahamat Hamit), betekora.hafiz@imsp-uac.org (Kora Hafiz Bete), ibrahima.mahamat@ugb.edu.sn (Barka Ibrahim Mahamat), mamadou-abdoul.diop@ugb.edu.sn (Mamadou Abdoul Diop)
}

doi: $10.22436 /$ jnsa.015.02.06

Received: 2021-06-19 Revised: 2021-11-01 Accepted: 2021-11-20 
time delay is critical when constructing realistic models in the domains of engineering, biology, and other sciences. In recent years, there has been a significant amount of interest in the examination of qualitative aspects of neutral stochastic differential equations, such as existence, uniqueness, and stability (see [11, 21, 30, 42, 45] and references therein). Lakhel [32] has shown the existence and uniqueness of mild solutions for a class of neutral stochastic functional differential evolution equations driven by a Rosenblatt process with changing time delays using a fixed point theorem.

The underlying notions in current mathematical theory known as controllability play a significant role both in deterministic and stochastic control problems, such as stabilization of unstable systems by feedback control. In recent years, the controllability problems for various linear and nonlinear deterministic and stochastic dynamical systems have been studied by employing diverse methodologies (see for example $[4,13,23,28,39,40,43,47,54,55]$ and the reference therein). For solving nonlinear problems (differential equations, stochastic differential equations, integrodifferential equations, and ...), it is generally recognized that fixed point theory is a valuable tool. Several scholars have contributed to the development of this theory by solving stochastic differential equations across multiple fixed point theorems. However, research on the controllability of impulsive neutral stochastic PDEs with delays and fractional Brownian motion $(\mathrm{fBm})$ is scarce. Ahmed examined approximate controllability of impulsive neutral stochastic functional differential equations with finite delay and $\mathrm{fBm}$ in Hilbert space in [2]. Cui and Yan [10] investigated controllability for fBm-driven neutral stochastic evolution equations with the Hurst parameter. Controllability of impulsive neutral stochastic functional differential equations with infinite delay driven by fBm in a real separable Hilbert space was studied by Boudaoui and Lakhel [5]. Chen [8] also addressed approximation control liability for semilinear stochastic equations driven by fBm using the Banach fixed point theorem.

Furthermore, stochastic functional differential equations with Poisson jumps have been increasingly popular in the modeling of phenomena that arise in a range of domains such as finance, economics, medicine, biology, and other related fields. In real life, it is usual for a stochastic system to transition from a normal state or a good state to a bad state, with the strength of the system being random. It is then obvious and required to incorporate a jump element in the stochastic differential equations. As a result, when analyzing the controllability of stochastic differential equations, it is crucial to evaluate the implications of Poisson leaps. We are pleased by the vast number of results on the controllability of stochastic differential equations with Poisson jumps that have recently been reported in the literature. So far, these themes have garnered a significant amount of attention, and there are a wealth of materials available on them. Lakhel et al. [33] showed the existence, uniqueness, and asymptotic behavior of mild solutions for a family of neutral functional stochastic differential equations with fBm and Poisson jumps. Huan and Agarwal [25] found attractive and quasi-invariant sets of the mild solution for impulsive neutral stochastic PDEs driven by Levy noise. Sakthivel and Ren [56], addressed the complete controllability of stochastic evolution equations with jumps in a separable Hilbert space, while in, Ren et al. [53] studied the approximate controllability of stochastic differential systems driven by Teugels martingales coupled with a Levy process. Huan and Gao [26] have expanded the conclusions of the study [24] for a class of nonlocal second-order impulsive neutral stochastic integrodifferential equations with indefinite delay and Poisson jumps. For further details concerning the stochastic PDEs with Poisson jumps, one can check the recent monograph [48] and the references therein.

In this paper, we consider the controllability results for impulsive neutral stochastic delay integrodifferential controls systems with delay driven by Rosenblatt process and Lévy noise of the form

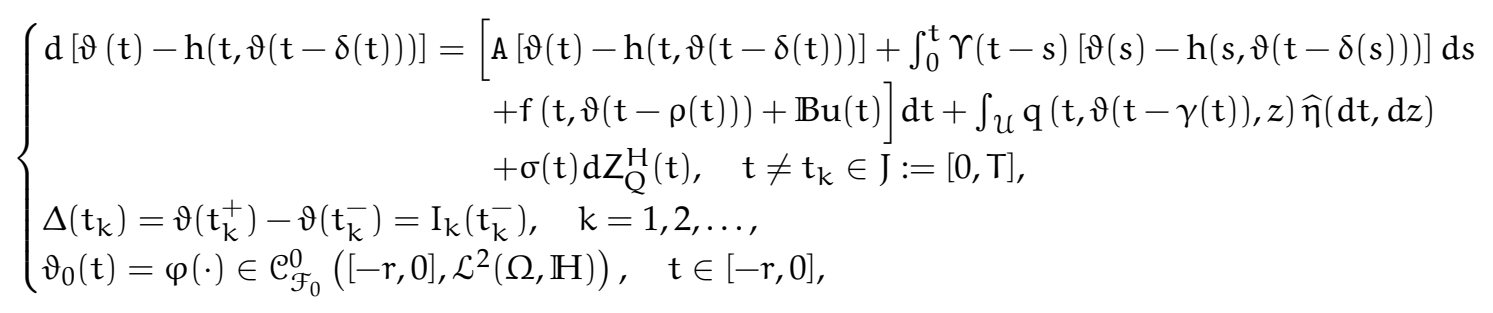

where $A: D(A) \subset \mathbb{H} \rightarrow \mathbb{H}$ is the infinitesimal generator of a $C_{0}$-semigroup $(S(t))_{t \geqslant 0}$ on Hilbert space 
$\mathbb{H}, \Upsilon(t)$ a closed linear operator on $\mathbb{H}$ with the domain $\mathrm{D}(\mathrm{A}) \subset \mathrm{D}(\Upsilon)$, which is independent of $t, t \geqslant 0$. The functions $\mathrm{h}, \mathrm{f}: \mathbb{R}^{+} \times \mathbb{H} \rightarrow \mathbb{H}, \sigma:[0, \infty) \rightarrow \mathcal{L}_{2}^{0}(\mathbb{Y}, \mathbb{H})$ and $\mathrm{q}: \mathbb{R}^{+} \times \mathbb{H} \times \mathcal{U} \rightarrow \mathbb{H}$ are Borel measurable. The functions $\delta, \rho, \gamma: \mathbb{R}^{+} \rightarrow[0, r]$ are continuous. $Z_{Q}^{H}$ is a Rosenblatt process with parameter $H \in(1 / 2,1)$ in a real and separable Hilbert space $\mathbb{Y}$. The control function $u$ takes values in $\mathrm{L}^{2}(\mathrm{~J}, \mathbb{U})$, the Hilbert space of admissible control functions for a separable Hilbert space $\mathbb{U}$ and $\mathbb{B}$ is a bounded linear operator from $\mathbb{U}$ into $\mathbb{H}, \mathrm{I}_{\mathrm{k}}: \mathbb{H} \rightarrow \mathbb{H}, \mathrm{k}=1,2, \ldots$ are appropriate functions. Furthermore, let $0=\mathrm{t}_{0}<\mathrm{t}_{1}<\cdots<\mathrm{t}_{\mathrm{k}}<\cdots$ be prefixed points, where $\vartheta\left(t_{k}^{+}\right)$and $\vartheta\left(t_{k}^{-}\right)$represent the right and left limits of $\vartheta(t)$ at $t=t_{k}$, respectively, and $\Delta\left(t_{k}\right)=\vartheta\left(t_{k}^{+}\right)-\vartheta\left(t_{k}^{-}\right)$, represents the jump of the function $\vartheta$ at time $t_{k}$ with $I_{k}$ determining the size of the jump.

However, to the best of authors knowledge the controllability of impulsive neutral stochastic delay integrodifferential controls systems with delay driven by Rosenblatt process and Lévy noise has not been investigated yet. Several researchers express the controllability results by the semigroup approach. The proposed work on the controllability of impulsive neutral stochastic delay integrodifferential controls systems with delay driven by Rosenblatt process and Lévy noise is new to the literature and more general result than the existing literature. The following are the most significant contributions and advantages of this article.

- Nonlinear impulsive neutral stochastic delay integrodifferential controls systems with Rosenblatt process and Lévy noise are developed.

- The fundamental advantage of the targeted technique is that it is based on resolvent operator theory in the sense of the Grimmer and Banach fixed point theorem, together with appropriate hypotheses.

- An example is provided in order to validate the theoretical conclusions that have been suggested.

The following is the overall structure of this study. In Section,2, we present a high-level overview of several fundamental notations, preliminaries, and assumptions. The results in Section 3 are devoted to the research of the controllability of the system (1.1), as well as their verification. Section 4 presents an illustration of the idea through the use of an example.

\section{Preliminaries}

Let $\left(\mathbb{H},\|\cdot\|_{\mathbb{H}},\langle\cdot, \cdot\rangle\right),\left(\mathbb{K},\|\cdot\|_{\mathbb{K}},\langle\cdot, \cdot\rangle\right)$ denote two real separable Hilbert spaces, with their vectors norms and their inner products, respectively. We denote by $\mathcal{L}(\mathbb{K}, \mathbb{H})$ the set of all linear bounded operators from $\mathbb{K}$ into $\mathbb{H}$, which is equipped with the usual operator norm $\|\cdot\|$. Let $\left(\Omega, \mathcal{F},\left\{\mathcal{F}_{t}\right\}_{t \in J}, \mathbb{P}\right)$ be a complete filtered probability space satisfying the usual condition (i.e., it is right continuous and $\mathcal{F}_{0}$ contains all $\mathbb{P}$ null sets). Let $\mathcal{L}(\mathbb{K}, \mathbb{H})$ represents the space of all bounded linear operators from $\mathbb{K}$ to $\mathbb{H}$ and $\mathrm{Q} \in$ $\mathcal{L}(\mathbb{K}, \mathbb{K})$ represents a non-negative self-adjoint operator. Let $\mathcal{L}_{2}^{0}=\mathrm{L}^{2}\left(\mathrm{Q}^{\frac{1}{2}} \mathbb{K}, \mathbb{H}\right)$ be the space of all HilbertSchmidt operators from $Q^{\frac{1}{2}} \mathbb{K}$ into $\mathbb{H}$, where $\mathcal{L}_{2}^{0}$ is a separable Hilbert space, equipped with the norm 1

$\|\Psi\|_{\mathcal{L}_{2}^{0}}^{2}=\left\|\Psi \mathrm{Q}^{\overline{2}}\right\|^{2}=\operatorname{Tr}\left(\Psi \mathrm{Q} \psi^{*}\right)$. Suppose that $\mathrm{p}(\mathrm{t}), \mathrm{t} \geqslant 0$ is $\sigma$-finite stationary $\mathcal{F}_{\mathrm{t}}$-adapted Poisson point process taking values in a measurable space $(\mathcal{U}, \mathcal{B}(\mathcal{U}))$.

\subsection{Rosenblatt process}

In this subsection, we recall some basic concepts on the Rosenblatt process as well as the Wiener integral with respect to it. Consider $\left(\xi_{n}\right)_{n \in Z}$ a stationary Gaussian sequence with mean zero and variance 1 such that its correlation function satisfies that $R(n):=\mathbb{E}\left(\xi_{0} \xi_{n}\right)=n^{\frac{2 H-2}{k}} L(n)$, with $H \in\left(\frac{1}{2}, 1\right)$ and $L$ is a slowly varying function at infinity. Let $g$ be a function of Hermite rank $k$, that is, if $g$ admits the following expansion in Hermite polynomials

$$
g(x)=\sum_{j \geqslant 0} c_{j} H_{j}(x), \quad c_{j}=\frac{1}{j !} \mathbb{E}\left(g\left(\xi_{0} H_{j}\left(\xi_{0}\right)\right)\right),
$$


then $k=\min \left\{\mathfrak{j} \mid \mathfrak{c}_{\mathfrak{j}} \neq 0\right\} \geqslant 1$, where $\mathrm{H}_{\mathfrak{j}}(x)$ is the Hermite polynomial of degree $\mathfrak{j}$ given by $\mathrm{H}_{\mathfrak{j}}(x)=$ $(-1)^{j} e^{\frac{x^{2}}{2}} \frac{d^{j}}{d x^{j}} e^{-\frac{x^{2}}{2}}$. Then, the Non-Central Limit Theorem (see, for example, Dobrushin and Major [15]) says $\frac{1}{n^{H}} \sum_{j=1}^{[n t]} g\left(\xi_{j}\right)$ converges as $n \rightarrow \infty$, in the sense of finite dimensional distributions, to the process

$$
\mathrm{Z}_{\mathrm{H}}^{\mathrm{k}}(\mathrm{t})=\mathrm{c}(\mathrm{H}, \mathrm{k}) \int_{\mathbb{R}^{\mathrm{k}}} \int_{0}^{\mathrm{t}}\left(\prod_{j=1}^{\mathrm{k}}\left(s-\mathrm{y}_{\mathrm{j}}\right)_{+}^{\left(-\frac{1}{2}+\frac{1-\mathrm{H}}{\mathrm{k}}\right)}\right) \mathrm{d} \mathrm{dB}\left(\mathrm{y}_{1}\right) \cdots \mathrm{dB}\left(\mathrm{y}_{\mathrm{k}}\right),
$$

where the above integral is a Wiener-Itô multiple integral of order $k$ with respect to the standard Brownian motion $(B(y))_{y \in \mathbb{R}}$ and $c(H, k)$ is a positive normalization constant depending only on $\mathrm{H}$ and $k$. The process $\left(Z_{\mathrm{H}}^{k}(t)\right)_{t \geqslant 0}$ is called as the Hermite process and it is $\mathrm{H}$ self-similar in the sense that for any $c>0$, $\left(Z_{\mathrm{H}}^{\mathrm{k}}(\mathrm{ct})\right) \stackrel{\mathrm{d}}{=}\left(\mathrm{c}^{\mathrm{H}} \mathrm{Z}_{\mathrm{H}}^{\mathrm{k}}(\mathrm{t})\right)$ and it has stationary increments.

The the fractional Brownian motion (which is obtained from $(2.1)$ when $k=1$ ) is the most used Hermite process for study evolution equations due to its large range of applications. When $k=2$ in (2.1), Taqqu [57] named the process as the Rosenblatt process. The stationarity of increments, self-similarity and long range dependence (see Tindel et al. [58]) were made that the Rosenblatt process is very important in practical applications. However, it is noted that Rosenblatt process is not Gaussian. In fact, due to their proprieties (long range dependence, self-similarity), the fractional Brownian motion process has large utilization in practical models, for instance in telecommunications and hydrology. So, many researchers prefer to use fractional Brownian motion than other processes because it is Gaussian and it facilitate calculations. However in concrete situations when the Gaussianity is not plausible for the model, one can use the Rosenblatt process. In recent years, there exist many works that investigated on diverse theoretical aspects of the Rosenblatt process. For example, Leonenko and Ahn [34] gave the rate of convergence to the Rosenblatt process in the non-central limit theorem and the wavelet-type expansion has been presented by Abry and Pipiras [1]. Tudor [60] established, the representation as a Wiener-Itô multiple integral with respect to the Brownian motion on a finite interval and developed the stochastic calculus with respect to it by using both pathwise type calculus and Malliavin calculus (see also Maejima and Tudor [36]). For more details for Rosenblatt process, we refer the reader to Maejima and Tudor [37, 38]), Pipiras and Taqqu [49] and the references therein.

Consider a time interval $[0, T]$ with arbitrary fixed horizon $T$ and let $\left\{Z_{H}(t), t \in[0, T]\right\}$ be a onedimensional Rosenblatt process with parameter $H \in\left(\frac{1}{2}, 1\right)$. According to the work of Tudor [60], the Rosenblatt process with parameter $\mathrm{H}>\frac{1}{2}$ can be written as

$$
Z_{H}(t)=d(H) \int_{0}^{t} \int_{0}^{t}\left[\int_{y_{1} \vee y_{2}}^{t} \frac{\partial K^{H^{\prime}}}{\partial u}\left(u, y_{1}\right) \frac{\partial K^{H^{\prime}}}{\partial u}\left(u, y_{2}\right) d u\right] d B\left(y_{1}\right) d B\left(y_{2}\right),
$$

where $\mathrm{K}^{\mathrm{H}}(\mathrm{t}, \mathrm{s})$ is given by

$$
K^{H}(t, s)=c_{H} s^{\frac{1}{2}-H} \int_{s}^{t}(u-s)^{H-3 / 2} u^{H-1 / 2} d u \text { for } t>s \text {, with } c_{H}=\sqrt{\frac{H(2 H-1)}{\beta\left(2-2 H, H-\frac{1}{2}\right)^{\prime}}},
$$

$\beta(.,$.$) denotes the Beta function, \mathrm{K}^{\mathrm{H}}(\mathrm{t}, \mathrm{s})=0$ when $\mathrm{t} \leqslant \mathrm{s},(\mathrm{B}(\mathrm{t}), \mathrm{t} \in[0, \mathrm{~T}])$ is a Brownian motion, $\mathrm{H}^{\prime}=$ $\frac{\mathrm{H}+1}{2}$ and $\mathrm{d}(\mathrm{H})=\frac{1}{\mathrm{H}+1} \sqrt{\frac{\mathrm{H}}{2(2 \mathrm{H}-1)}}$ is a normalizing constant. The covariance of the Rosenblatt process $\left\{Z_{H}(t), t \in[0, T]\right\}$ satisfies

$$
\mathbf{E}\left(Z_{\mathrm{H}}(\mathrm{t}) \mathrm{Z}_{\mathrm{H}}(\mathrm{s})\right)=\frac{1}{2}\left(\mathrm{~s}^{2 \mathrm{H}}+\mathrm{t}^{2 \mathrm{H}}-|\mathrm{s}-\mathrm{t}|^{2 \mathrm{H}}\right) .
$$

The covariance structure of the Rosenblatt process allows to construct Wiener integral with respect to it. We refer to Maejima and Tudor [36] for the definition of Wiener integral with respect to general Hermite processes and to Kruk et al. [31] for a more general context (see also Tudor [60]). Note that

$$
Z_{H}(t)=\int_{0}^{T} \int_{0}^{T} I\left(1_{[0, t]}\right)\left(y_{1}, y_{2}\right) d B\left(y_{1}\right) d B\left(y_{2}\right)
$$


where the operator I is defined on the set of functions $f:[0, T] \rightarrow \mathbb{R}$, which takes its values in the set of functions $\mathrm{g}:[0, \mathrm{~T}]^{2} \rightarrow \mathbb{R}^{2}$ and is given by

$$
I(f)\left(y_{1}, y_{2}\right)=d(H) \int_{y_{1} \vee y_{2}}^{T} f(u) \frac{\partial K^{H^{\prime}}}{\partial u}\left(u, y_{1}\right) \frac{\partial K^{H^{\prime}}}{\partial u}\left(u, y_{2}\right) d u
$$

Let $f$ be an element of the set $\mathcal{E}$ of step functions on $[0, T]$ of the form

$$
f=\sum_{i=0}^{n-1} a_{i} 1_{\left(t_{i}, t_{i+1}\right]}, t_{i} \in[0, T] .
$$

Then, it is natural to define its Wiener integral with respect to $Z_{H}$ as

$$
\int_{0}^{T} f(u) d Z_{H}(u):=\sum_{i=0}^{n-1} a_{i}\left(Z_{H}\left(t_{i+1}\right)-Z_{H}\left(t_{i}\right)\right)=\int_{0}^{T} \int_{0}^{T} I(f)\left(y_{1}, y_{2}\right) d B\left(y_{1}\right) d B\left(y_{2}\right) .
$$

Let $\mathcal{H}$ be the set of functions $f$ such that

$$
\|f\|_{\mathcal{H}}^{2}:=2 \int_{0}^{T} \int_{0}^{T}\left(I(f)\left(y_{1}, y_{2}\right)\right)^{2} d y_{1} d y_{2}<\infty
$$

It follows that (see Tudor[60])

$$
\|f\|_{\mathcal{H}}^{2}=H(2 H-1) \int_{0}^{T} \int_{0}^{T} f(u) f(v)|u-v|^{2 H-2} d u d v .
$$

It has been proved in Maejima and Tudor [36] that the mapping $f \longmapsto \int_{0}^{T} f(u) d Z_{H}(u)$ defines an isometry from $\mathcal{E}$ to $L^{2}(\Omega)$ and it can be extended continuously to an isometry from $\mathcal{H}$ to $L^{2}(\Omega)$ because $\mathcal{E}$ is dense in $\mathcal{H}$. We call this extension as the Wiener integral of $f \in \mathcal{H}$ with respect to $Z_{\mathrm{H}}$. It is noted that the space $\mathcal{H}$ contains not only functions but its elements could be also distributions. Therefore it is suitable to know subspaces $|\mathcal{H}|$ of $\mathcal{H}:|\mathcal{H}|=\left\{f:[0, \mathrm{~T}] \rightarrow \mathbb{R}\left|\int_{0}^{\mathrm{T}} \int_{0}^{\mathrm{T}}\right| \mathrm{f}(\mathrm{u})|| \mathrm{f}(v)|\mathrm{u}-v|^{2 \mathrm{H}-2} \mathrm{~d} u \mathrm{~d} v<\infty\right\}$. The space $|\mathcal{H}|$ is not complete with respect to the norm $\|\cdot\|_{\mathcal{H}}$ but it is a Banach space with respect to the norm

$$
\|f\|_{|\mathcal{H}|}^{2}=\mathrm{H}(2 \mathrm{H}-1) \int_{0}^{T} \int_{0}^{T}|\mathrm{f}(\mathrm{u}) \| \mathrm{f}(v)| \mathrm{u}-\left.v\right|^{2 \mathrm{H}-2} \mathrm{~d} u \mathrm{~d} v .
$$

As a consequence, we have

$$
\mathrm{L}^{2}([0, \mathrm{~T}]) \subset \mathrm{L}^{1 / \mathrm{H}}([0, \mathrm{~T}]) \subset|\mathcal{H}| \subset \mathcal{H} .
$$

For any $f \in \mathrm{L}^{2}([0, \mathrm{~T}])$, we have

$$
\|f\|_{|\mathcal{H}|}^{2} \leqslant 2 \mathrm{HT}^{2 \mathrm{H}-1} \int_{0}^{\mathrm{T}}|\mathrm{f}(\mathrm{s})|^{2} \mathrm{ds} \text { and }\|\mathrm{f}\|_{|\mathcal{H}|}^{2} \leqslant \mathrm{C}(\mathrm{H})\|\mathrm{f}\|_{\mathrm{L}^{1 / \mathrm{H}}([0, \mathrm{~T}])^{\prime}}^{2}
$$

for some constant $\mathrm{C}(\mathrm{H})>0$. Let $\mathrm{C}(\mathrm{H})>0$ stands for a positive constant depending only on $\mathcal{H}$ and its value may be different in different appearances. Define the linear operator $\mathrm{K}_{\mathrm{H}}^{*}$ from $\mathcal{E}$ to $\mathrm{L}^{2}([0, \mathrm{~T}])$ by

$$
\left(K_{H}^{*} f\right)\left(y_{1}, y_{2}\right)=\int_{y 1 \vee y_{2}}^{T} f(t) \frac{\partial \mathcal{K}}{\partial t}\left(t, y_{1}, y_{2}\right) d t,
$$

where $\mathcal{K}$ is the kernel of Rosenblatt process in representation (2.2)

$$
\mathcal{K}\left(t, y_{1}, y_{2}\right)=1_{[0, t]}\left(y_{1}\right) 1_{[0, t]}\left(y_{2}\right) \int_{y_{1} \vee y_{2}}^{t} \frac{\partial K^{H^{\prime}}}{\partial u}\left(u, y_{1}\right) \frac{\partial K^{H^{\prime}}}{\partial u}\left(u, y_{2}\right) d u
$$


Note that $\left(K_{H}^{*} 1_{[0, t]}\right)\left(y_{1}, y_{2}\right)=\mathcal{K}\left(t, y_{1}, y_{2}\right) 1_{[0, t]}\left(y_{1}\right) 1_{[0, t]}\left(y_{2}\right)$. The operator $K_{H}^{*}$ is an isometry between $\mathcal{E}$ to $\mathrm{L}^{2}([0, \mathrm{~T}])$, which can be extended to the Hilbert space $\mathcal{H}$. In fact, for any $s, t \in[0, T]$ we have

$$
\begin{aligned}
\left\langle\mathrm{K}_{\mathrm{H}}^{*} 1_{[0, \mathrm{t}]}, \mathrm{K}_{\mathrm{H}}^{*} 1_{[0, s]}\right\rangle_{\mathrm{L}^{2}([0, \mathrm{~T}])} & =\left\langle\mathcal{K}(\mathrm{t}, . . .) 1_{[0, \mathrm{t}]}, \mathcal{K}(\mathrm{s}, . . .) 1_{[0, s]}\right\rangle_{\mathrm{L}^{2}([0, \mathrm{~T}])} \\
& =\int_{0}^{\mathrm{t} \wedge \mathrm{s}} \int_{0}^{\mathrm{t} \wedge \mathrm{s}} \mathcal{K}\left(\mathrm{t}, \mathrm{y}_{1}, \mathrm{y}_{2}\right) \mathcal{K}\left(\mathrm{s}, \mathrm{y}_{1}, \mathrm{y}_{2}\right) \mathrm{d} \mathrm{y}_{1} \mathrm{~d} \mathrm{y}_{2} \\
& =\mathrm{H}(2 \mathrm{H}-1) \int_{0}^{\mathrm{t}} \int_{0}^{s}|\mathrm{u}-v|^{2 \mathrm{H}-2} \mathrm{dud} v \\
& =\left\langle 1_{[0, \mathrm{t}]}, 1_{[0, \mathrm{~s}]}\right\rangle_{\mathcal{H}} .
\end{aligned}
$$

Moreover, for $f \in \mathcal{H}$, we have

$$
Z_{H}(f)=\int_{0}^{T} \int_{0}^{T}\left(K_{H}^{*} f\right)\left(y_{1}, y_{2}\right) d B\left(y_{1}\right) d B\left(y_{2}\right)
$$

Let $\left\{Z_{\mathfrak{n}}(t)\right\}_{\mathfrak{n} \in \mathbb{N}}$ be a sequence of two-sided one dimensional Rosenblatt process mutually independent on $(\Omega, \mathcal{F}, \mathbb{P})$. We consider a $\mathrm{K}$-valued stochastic process $Z_{Q}(t)$ given by the following series

$$
\mathrm{Z}_{\mathrm{Q}}(\mathrm{t})=\sum_{\mathrm{n}=1}^{\infty} z_{\mathrm{n}}(\mathrm{t}) \mathrm{Q}^{1 / 2} e_{\mathrm{n}}, \quad \mathrm{t} \geqslant 0
$$

Moreover, if $\mathrm{Q}$ is a non-negative self-adjoint trace class operator, then this series converges in the space $K$, that is, it holds that $Z_{Q}(t) \in L^{2}(\Omega, K)$. Then, we say that the above $Z_{Q}(t)$ is a $K$-valued Q-Rosenblatt process with covariance operator $Q$. For instance, if $\left\{\sigma_{n}\right\}_{n \in \mathbb{N}}$ is a bounded sequence of non-negative real numbers such that $Q e_{n}=\sigma_{n} e_{n}$, by assuming that $Q$ is a nuclear operator in $K$, then the stochastic process

$$
\mathrm{Z}_{\mathrm{Q}}(\mathrm{t})=\sum_{\mathrm{n}=1}^{\infty} z_{\mathrm{n}}(\mathrm{t}) \mathrm{Q}^{1 / 2} e_{\mathrm{n}}=\sum_{\mathrm{n}=1}^{\infty} \sqrt{\sigma_{\mathrm{n}}} z_{\mathrm{n}}(\mathrm{t}) e_{\mathrm{n}}, \quad \mathrm{t} \geqslant 0,
$$

is well-defined as a K-valued Q-Rosenblatt process.

Definition 2.1 (Tudor [60]). Let $\varphi:[0, \mathrm{~T}] \rightarrow \mathcal{L}_{2}^{0}$ such that $\sum_{n=1}^{\infty}\left\|\mathrm{K}_{\mathrm{H}}^{*}\left(\varphi \mathrm{Q}^{1 / 2} e_{n}\right)\right\|_{\mathrm{L}^{2}([0, T] ; \mathcal{H})}<\infty$. Then, its stochastic integral with respect to the Rosenblatt process $Z_{Q}(t)$ is defined, for $t \geqslant 0$, as follows:

$$
\int_{0}^{t} \varphi(s) d Z_{Q}(s):=\sum_{n=1}^{\infty} \int_{0}^{t} \varphi(s) Q^{1 / 2} e_{n} d z_{n}(s)=\sum_{n=1}^{\infty} \int_{0}^{t} \int_{0}^{t}\left(K_{H}^{*}\left(\varphi Q^{1 / 2} e_{n}\right)\right)\left(y_{1}, y_{2}\right) d B\left(y_{1}\right) d B\left(y_{2}\right) .
$$

Lemma 2.2. For $\psi:[0, \mathrm{~T}] \rightarrow \mathcal{L}_{2}^{0}$ such that $\sum_{n=1}^{\infty}\left\|\psi \mathrm{Q}^{1 / 2} e_{\mathrm{n}}\right\|_{\mathrm{L}^{1 / \mathrm{H}}([0, \mathrm{~T}] ; \mathrm{U})}<\infty$ holds, and for any $\mathrm{a}, \mathrm{b} \in[0, \mathrm{~T}]$ with $\mathrm{b}>\mathrm{a}$, we have

$$
\mathbf{E}\left\|\int_{a}^{b} \psi(s) d Z_{Q}(s)\right\|^{2} \leqslant c(H)(b-a)^{2 H-1} \sum_{n=1}^{\infty} \int_{a}^{b}\left\|\psi(s) Q^{1 / 2} e_{n}\right\|^{2} d s .
$$

If, in addition,

$$
\sum_{n=1}^{\infty}\left\|\psi(t) Q^{1 / 2} e_{n}\right\| \text { is uniformly convergent for } \mathrm{t} \in[0, \mathrm{~T}]
$$

then, it holds that

$$
\mathbf{E}\left\|\int_{a}^{b} \psi(s) d Z_{Q}(s)\right\|^{2} \leqslant C(H)(b-a)^{2 H-1} \int_{a}^{b}\|\psi(s)\|_{\mathcal{L}_{2}^{0}}^{2} d s
$$


Proof. Let $\left\{\boldsymbol{e}_{\mathfrak{n}}\right\}_{\mathfrak{n} \in \mathbb{N}}$ be the complete orthogonal basis of $\mathbb{K}$ introduced above. Applying (2.3) and Hölder inequality, we have

$$
\begin{aligned}
\mathbf{E}\left\|\int_{a}^{b} \psi(s) d Z_{Q}(s)\right\|^{2} & =\mathbf{E}\left\|\sum_{n=1}^{\infty} \int_{a}^{b} \psi(s) Q^{1 / 2} e_{n} d z_{n}(s)\right\|^{2} \\
& =\sum_{n=1}^{\infty} \mathbf{E}\left\|\int_{a}^{b} \psi(s) Q^{1 / 2} e_{n} d z_{n}(s)\right\|^{2} \\
& =\sum_{n=1}^{\infty} H(2 H-1) \int_{a}^{b} \int_{a}^{b}\left\|\psi(s) Q^{1 / 2} e_{n}\right\|\left\|\psi(t) Q^{1 / 2} e_{n}\right\||t-s|^{2 H-2} d s d t \\
& \leqslant C(H) \sum_{n=1}^{\infty}\left(\int_{a}^{b}\left\|\psi(s) Q^{1 / 2} e_{n}\right\|^{1 / H} d s\right)^{2 H} \\
& \leqslant C(H)(b-a)^{2 H-1} \sum_{n=1}^{\infty} \int_{a}^{b}\left\|\psi(s) Q^{1 / 2} e_{n}\right\|^{2} d s .
\end{aligned}
$$

Let $\mathbb{Y}$ be a separable Hilbert space. Let $p(t), t \geqslant 0$ be $\sigma$-finite stationary $\mathcal{F}_{t}$-adapted $\mathbb{Y}$-valued Poisson point process. Then for any $\mathrm{F} \in \mathcal{B}(\mathbb{Y}-\{0\})$, which denotes the Borel $\sigma$-field of $(\mathbb{Y}-\{0\})$, where $0 \notin \overline{\mathrm{F}}$, we get a counting Poisson random measure $\eta$ on $(\mathbb{Y}-\{0\})$ :

$$
\begin{aligned}
\eta((0, t) \times F) & :=\sum_{0<s \leqslant t} 1_{F}(p(s))=\#\{0<s \leqslant t, p(s) \in F\}, \\
\eta\left(\left(t_{1}, t_{2}\right] \times F\right): & =\eta\left(\left(0, t_{2}\right) \times F\right)-\eta\left(\left(0, t_{1}\right) \times F\right) .
\end{aligned}
$$

We shall denote $\eta(t, F):=\eta((0, t) \times F)$. Then, it is known that there exists a $\sigma$-finite measure $\lambda$ such that

$$
\mathbf{E}[\eta(\mathrm{t}, \mathrm{F})]=\lambda(\mathrm{F}) \mathrm{t}, \quad \quad \mathbb{P}[\eta(\mathrm{t}, \mathrm{F})=\mathrm{n}]=\frac{\exp \left(-\mathrm{t} \lambda(\mathrm{F})(\lambda(\mathrm{F}) \mathrm{t})^{\mathrm{n}}\right)}{\mathrm{n} !} .
$$

The measure $\lambda$ is called the Lévy measure. Then, $t>0$, the measure $\widehat{\eta}$ is defined by

$$
\widehat{\eta}([0, t], F)=\eta([0, t], F)-t \lambda(F) .
$$

The measure $\widehat{\eta}(d t, d y)$ is called the compensated Poisson random measure and $\lambda(F)$ is called the compensator.

Let $\mathcal{U} \in \mathcal{B}(\mathbb{Y}-\{0\})$, where $0 \notin$ the closure of $\mathcal{U}$. Let $\lambda_{\mathcal{U}}$ denotes the restriction of the measure $\lambda$ to $\mathcal{U}$ still denoted by $\lambda$, such that $\lambda$ is finite on $\mathcal{U}$. Denote by $\mathcal{P}^{2}([0, t] \times \mathcal{U} ; \mathbb{H})$ the space of all predictable mappings $\mathrm{k}:[0, \mathrm{t}] \times \mathcal{U} \rightarrow \mathbb{H}$ for which

$$
\int_{0}^{t} \int_{\mathcal{U}} \mathbf{E}\|\kappa(s, y)\|_{\mathbb{H}}^{2} \lambda(d y) d s<\infty
$$

We may then define the $\mathbb{H}$-valued stochastic integral

$$
\int_{0}^{t} \int_{U} \kappa(s, y) \widehat{\eta}(d s, d y):=\int_{0}^{t} \int_{U} \kappa(s, y) \eta(d s, d y)-\int_{0}^{t} \int_{U} \kappa(s, y) \lambda(d y) d s,
$$

where

$$
\int_{0}^{t} \int_{\mathcal{U}} \kappa(s, y) \eta(d s, d y):=\sum_{0<s \leqslant t} \kappa(s, p(s)) 1_{u}(p(s)) .
$$


Furthermore, we can see that $\int_{0}^{t} \int_{u} k(s, y) \widehat{\eta}(d s, d y)$ is an $\mathbb{H}$-valued centered square integrable martingale such that

$$
\mathbf{E}\left(\left\|\int_{0}^{t} \int_{\mathcal{U}} \kappa(s, y) \widehat{\eta}(d s, d y)\right\|_{\mathbb{H}}^{2}\right)=\int_{0}^{t} \int_{\mathcal{U}} \mathbf{E}\|\kappa(s, y)\|_{\mathbb{H}}^{2} \lambda(d y) d s .
$$

We can refer to Protter [50] for a systematic theory about stochastic integrals of this kind.

\subsection{Partial integrodifferential equations in Banach spaces}

In this section, we recall some fundamental results needed to establish our main results. For the theory of resolvent operators we refer the reader to [19]. Throughout this paper, $\mathbb{X}$ is a Banach space, $A$ and $\Upsilon(t)$ are closed linear operators on $\mathbb{X}$. Y represents the Banach space $D(A)$ equipped with the graph norm defined by

$$
|y|_{Y}:=|A y|+|y| \text { for } y \in Y .
$$

The notations $C([0,+\infty) ; Y), \mathcal{B}(Y, \mathbb{X})$ stand for the space of all continuous functions from $[0,+\infty)$ into $Y$, the set of all bounded linear operators from $Y$ into $\mathbb{X}$, respectively. We consider the following Cauchy problem

$$
\left\{\begin{array}{l}
\vartheta^{\prime}(t)=A \vartheta(t)+\int_{0}^{t} \curlyvee(t-s) \vartheta(s) d s, \text { for } t \geqslant 0, \\
\vartheta(0)=\vartheta_{0} \in \mathbb{X} .
\end{array}\right.
$$

Definition 2.3 ([19]). A resolvent operator for Eq. (2.4) is a bounded linear operator valued function $R(t) \in \mathcal{L}(\mathbb{X})$ for $t \geqslant 0$, satisfying the following properties:

(i) $\mathrm{R}(0)=\mathrm{I}$ and $|\mathrm{R}(\mathrm{t})| \leqslant M e^{\beta t}$ for some constants $M$ and $\beta$;

(ii) for each $x \in \mathbb{X}, R(t) x$ is strongly continuous for $t \geqslant 0$;

(iii) $\mathrm{R}(\mathrm{t}) \in \mathcal{L}(\mathrm{Y})$ for $\mathrm{t} \geqslant 0$. For $\vartheta \in \mathrm{Y}, \mathrm{R}(\cdot) \vartheta \in \mathrm{C}^{1}([0,+\infty) ; \mathbb{X}) \cap \mathrm{C}([0,+\infty) ; \mathrm{Y})$ and

$$
R^{\prime}(t) \vartheta=A R(t) \vartheta+\int_{0}^{t} \curlyvee(t-s) R(s) \vartheta d s=R(t) A \vartheta+\int_{0}^{t} R(t-s) \Upsilon(s) \vartheta d s \quad \text { for } t \geqslant 0 .
$$

For additional details on resolvent operators, we refer the reader to [19]. To deal with the existence of a resolvent operator we introduce the following assumptions.

$\left(\mathbf{H}_{1}\right)$ A is the infinitesimal generator of a strongly continuous semigroup $\{S(t)\}_{t} \geqslant 0$ on $\mathbb{X}$.

$\left(\mathbf{H}_{2}\right)$ For all $t \geqslant 0, \Upsilon(t)$ is a closed linear operator from $D(A)$ to $\mathbb{X}$, and $\Upsilon(t) \in \mathcal{B}(Y, \mathbb{X})$. For any $y \in Y$, the map $t \rightarrow \Upsilon(t) y$ is bounded, differentiable and the derivative $t \rightarrow \Upsilon^{\prime}(t) y$ is bounded and uniformly continuous on $\mathbb{R}^{+}$.

Theorem 2.4 ([19, Theorem 3.7]). Assume that $\left(\mathbf{H}_{1}\right)-\left(\mathbf{H}_{2}\right)$ hold. Then there exists a unique resolvent operator for the Cauchy problem (2.4).

We denote by $\mathcal{C}_{\mathrm{T}}:=\mathcal{C}\left([-r, T], \mathcal{L}^{2}(\Omega, \mathbb{H})\right)$ the Banach space of all continuous functions from from $[-r, T]$ into $\mathcal{L}^{2}(\Omega, \mathbb{H})$ such that for all $\vartheta \in \mathcal{C}_{\mathrm{T}}$,

$$
\|\vartheta\|_{\mathcal{C}_{\mathrm{T}}}:=\sup _{\mathrm{t} \in[-\mathrm{r}, \mathrm{T}]}\left(\mathbf{E}\|\vartheta(\mathrm{t})\|^{2}\right)^{\frac{1}{2}}
$$

and let us consider the set $\mathcal{B}_{\mathrm{T}}=\left\{\vartheta \in \mathcal{C}_{\mathrm{T}}: \vartheta(s)=\varphi(s), \forall s \in[-r, 0]\right\}$. The set $\mathcal{B}_{\mathrm{T}}$ is a closed subset of $\mathcal{C}_{\mathrm{T}}$ endowed with norm $\|\cdot\|_{\mathfrak{C}_{\mathrm{T}}}$. Then, $\mathcal{C}_{\mathrm{T}}$ and $\mathcal{B}_{\mathrm{T}}$ with the above norm are Banach spaces. Let $\mathcal{C}_{\mathcal{F}_{0}}^{0}\left([-r, 0], \mathcal{L}^{2}(\Omega, \mathbb{H})\right)$ denote the family of all bounded $\mathcal{F}_{0}\left(\mathcal{F}_{\mathfrak{t}}\right)$-measurable, $\mathcal{C}_{0}:=\mathcal{C}\left([-r, 0], \mathcal{L}^{2}(\Omega, \mathbb{H})\right)$ valued random variables $\varphi$, satisfying

$$
\|\varphi\|_{e_{0}}:=\sup _{\mathbf{t} \in[-r, 0]}\left(\mathbf{E}\|\varphi(\mathbf{t})\|^{2}\right)^{\frac{1}{2}}<\infty .
$$

Now, we give the definition of mild solution for (1.1). 
Definition 2.5. A càdlàg stochastic process $\vartheta:[-r, T] \rightarrow \mathbb{H}, 0 \leqslant T<\infty$ is called a mild solution of (1.1) on $[-r, T]$ if $\vartheta_{0}(\cdot)=\varphi \in \mathcal{C}_{\mathcal{F}_{0}}^{0}\left([-r, 0], \mathcal{L}^{2}(\Omega, \mathbb{H})\right)$ on $[-r, 0]$ a.s., and for each $t \geqslant 0$ the following conditions hold:

(i) $\vartheta(t)$ is $\mathcal{F}_{t}$-adapted;

(ii) $\vartheta(t)$ satisfies the following integral equation:

$$
\begin{aligned}
\vartheta(t)= & R(t)[\varphi(0)-h(0, \varphi(-\delta(0)))]+h(t, \vartheta(t-\delta(t)))+\int_{0}^{t} R(t-s) f(s, \vartheta(s-\rho(s))) d s \\
& +\int_{0}^{t} R(t-s) \mathbb{B} U(s) d s+\int_{0}^{t} \int_{u} R(t-s) q(s, \vartheta(s-\gamma(s)), z) \widehat{\eta}(d s, d z) \\
& +\int_{0}^{t} R(t-s) \sigma(s) d Z_{Q}^{H}(s)+\sum_{0<t_{k}<t} R\left(t-t_{k}\right) I_{k}\left(\vartheta\left(t_{k}^{-}\right)\right), \mathbb{P}, \text { a.s. }
\end{aligned}
$$

Definition 2.6. The stochastic integrodifferential equations (1.1) is said to be controllable on the interval $[-r, T]$, if for every initial stochastic process $\vartheta(\cdot)=\varphi$ defined on $[-r, 0]$, there exists a stochastic control $\mathrm{u} \in \mathcal{L}^{2}(\mathrm{~J}, \mathbb{U})$ which is adapted to the filtration $\left\{\mathcal{F}_{t}\right\}_{\mathrm{t} \in \mathrm{J}}$ such that the solution $\vartheta(\cdot)$ of the system (1.1) satisfies $\vartheta(T)=\vartheta_{1}$, where $\vartheta_{1}$ and $T$ are preassigned the terminal state and time, respectively.

\section{Main results}

In order to prove the existence and controllability results, one need to assume the following hypotheses hold.

$\left(\mathbf{H}_{3}\right)$ The resolvent operator associated with equation (2.4) is exponentially stable. That is: there exit some constants $\mu_{0}>0, \tilde{M}>0$, such that

$$
\|\mathrm{R}(\mathrm{t})\| \leqslant \tilde{M} e^{-\mu_{0} t}
$$

$\left(\mathbf{H}_{4}\right)$ The function $\mathrm{f}: \mathrm{J} \times \mathbb{H} \rightarrow \mathbb{H}$ satisfies : there exist positive constants $\mathrm{C}_{\mathrm{h}}>0$ and $\mathrm{M}_{\mathrm{h}}>0$ such that for all $t \geqslant 0$ and $x, y \in \mathbb{H}$

$$
\|h(t, x)-h(t, y)\| \leqslant C_{h}\|x-y\|, \text { and }\|h(t, x)\|^{2} \leqslant M_{h}\left(1+\|x\|^{2}\right) .
$$

$\left(\mathbf{H}_{5}\right)$ For all $t \geqslant 0, x, y \in \mathbb{H}, z \in \mathcal{U}$, the functions $\mathrm{f}$, q satisfy: there exist constants $\mathrm{C}_{\mathrm{f}}>0, \mathrm{C}_{\mathrm{q}}>0, \mathrm{M}_{\mathrm{f}}>$ $0, M_{\mathrm{q}}>0$ such that

$$
\|f(t, x)-f(t, y)\| \leqslant C_{f}\|x-y\|, \quad\|f(t, x)\|^{2} \leqslant M_{f}\left(1+\|x\|^{2}\right),
$$

and

$$
\int_{u}\|\mathrm{q}(\mathrm{t}, x, z)-\mathrm{q}(\mathrm{t}, \mathrm{y}, z)\|^{2} v(\mathrm{~d} z) \leqslant \mathrm{C}_{\mathrm{q}}^{2}\|x-y\|^{2}, \quad \int_{\mathcal{u}}\|\mathrm{q}(\mathrm{t}, \mathrm{y}, z)\|^{2} v(\mathrm{~d} z) \leqslant \mathrm{M}_{\mathrm{q}}\left(1+\|\mathrm{y}\|^{2}\right) .
$$

$\left(\mathbf{H}_{6}\right)$ The functions $\mathrm{I}_{k} \in \mathcal{C}(\mathbb{H}, \mathbb{H}), k=1,2 \ldots$, satisfy the following conditions: there exist some positive constants $C_{I_{k}}, M_{I_{k}}$ such that for all $x, y \in \mathbb{H}$,

$$
\left\|I_{k}(x)-I_{k}(y)\right\| \leqslant C_{I_{k}}\|x-y\|, \quad\left\|I_{k}(x)\right\|^{2} \leqslant d_{k}\left(1+\|x\|^{2}\right), \quad \sum_{k=1}^{\infty} C_{I_{k}}<\infty, \quad \sum_{k=1}^{\infty} d_{k}<\infty .
$$

$\left(\mathbf{H}_{7}\right)$ The function $\mathrm{h}$ is continuous in the quadratic mean sense: for all $\vartheta \in \mathcal{C}\left(\mathrm{J}, \mathcal{L}^{2}(\Omega, \mathbb{H})\right)$,

$$
\lim _{t \rightarrow s} E\|h(t, \vartheta(t))-h(s, \vartheta(s))\|^{2}=0 .
$$


$\left(\mathbf{H}_{8}\right)$ The function $\sigma:[0, \infty) \rightarrow \mathcal{L}_{2}^{0}(\mathbb{Y}, \mathbb{H})$ satisfies the following condition:

$$
\int_{0}^{t} e^{\mu_{0} s}\|\sigma(s)\|_{\mathcal{L}_{2}^{0}}^{2} \mathrm{~d} s<\infty, \forall t>0
$$

$\left(\mathbf{H}_{9}\right) \mathbb{B}: \mathbb{U} \rightarrow \mathbb{H}$ is bounded linear operator and the operator $\Gamma: \mathrm{L}^{2}(\mathrm{~J}, \mathbb{U}) \rightarrow \mathrm{L}^{2}(\Omega, \mathbb{H})$ defined by

$$
\Gamma \mathrm{u}=\int_{0}^{\mathrm{b}} \mathrm{R}(\mathrm{t}-\mathrm{s}) \mathrm{Bu}(\mathrm{s}) \mathrm{ds}
$$

has an inverse operator $\Gamma^{-1}$ which takes values in $L^{2}(J, \mathbb{U}) \backslash \operatorname{Ker} \Gamma$, where the kernel space of $\Gamma$ is defined by $\operatorname{Ker} \Gamma=\left\{x \in \mathrm{L}^{2}(\mathrm{~J}, \mathbb{U}): \Gamma x=0\right\}$ (see $[51,59]$ ) and there exist two positive constants $M_{\mathbb{B}}$ and $M_{\Gamma}$ such that

$$
\|\mathbb{B}\|^{2} \leqslant M_{\mathbb{B}},\left\|\Gamma^{-1}\right\|^{2} \leqslant M_{\Gamma} .
$$

Theorem 3.1. If hypotheses $\left(\mathbf{H}_{1}\right)-\left(\mathbf{H}_{9}\right)$ hold and $\vartheta_{0} \in \mathbb{H}$, then, the impulsive stochastic integrodifferential system (1.1) is controllable on $[-r, \mathrm{~T}]$ provided that

$$
\mathrm{C}_{\mathrm{h}}^{2}+\tilde{M}^{2}\left(\sum_{\mathrm{k}=1}^{+\infty} \mathrm{C}_{\mathrm{I}_{\mathrm{k}}}\right)^{2}<\frac{1}{5}
$$

Proof. Using the hypothesis $\left(\mathbf{H}_{9}\right)$, we define the control $\mathrm{u}(\cdot)$ for an arbitrary $\vartheta(\cdot)$ by

$$
\begin{aligned}
\mathrm{u}(\mathrm{t})= & \Gamma^{-1}\left\{\vartheta_{1}-\mathrm{R}(\mathrm{T})[\varphi(0)-\mathrm{h}(0, \varphi(-\delta(0)))]-\mathrm{h}(\mathrm{T}, \vartheta(T-\delta(T)))-\int_{0}^{T} \mathrm{R}(\mathrm{T}-\mathrm{s}) \mathrm{f}(\mathrm{s}, \vartheta(s-\rho(s))) \mathrm{d} s\right. \\
& -\int_{0}^{T} \int_{\mathcal{U}} \mathrm{R}(\mathrm{T}-\mathrm{s}) \mathrm{q}(\mathrm{s}, \vartheta(\mathrm{s}-\gamma(\mathrm{s})), z) \widehat{\eta}(\mathrm{ds}, \mathrm{d} z)-\int_{0}^{T} \mathrm{R}(\mathrm{T}-\mathrm{s}) \sigma(\mathrm{s}) \mathrm{d} \mathrm{Z}_{\mathrm{Q}}^{\mathrm{H}}(\mathrm{s}) \\
& \left.-\sum_{0<\mathrm{t}_{\mathrm{k}}<\mathrm{T}} \mathrm{R}\left(\mathrm{T}-\mathrm{t}_{\mathrm{k}}\right) \mathrm{I}_{\mathrm{k}}\left(\vartheta\left(\mathrm{t}_{\mathrm{k}}^{-}\right)\right)\right\}(\mathrm{t}) .
\end{aligned}
$$

We transform (1.1) into a fixed point problem. By using the above control, we show that the operator $\Psi: \mathcal{B}_{\mathrm{T}} \rightarrow \mathcal{B}_{\mathrm{T}}$ defined by $(\Psi \vartheta)(\mathrm{t})=\varphi(\mathrm{t}), \mathrm{t} \in[-\mathrm{r}, 0]$ and $\forall \mathrm{t} \in \mathrm{J}$,

$$
\begin{aligned}
& (\Psi \vartheta)(\mathrm{t})=\mathrm{R}(\mathrm{t})[\varphi(0)-\mathrm{h}(0, \varphi(-\delta(0)))]+\mathrm{h}(\mathrm{t}, \vartheta(\mathrm{t}-\delta(\mathrm{t})))+\int_{0}^{\mathrm{t}} \mathrm{R}(\mathrm{t}-\mathrm{s}) \mathrm{f}(\mathrm{s}, \vartheta(s-\rho(s))) \mathrm{d} s \\
& +\int_{0}^{t} \int_{\mathcal{U}} R(t-s) q(s, \vartheta(s-\gamma(s)), z) \widehat{\eta}(d s, d z)+\int_{0}^{t} R(t-s) \sigma(s) d Z_{Q}^{H}(s) \\
& +\sum_{0<t_{k}<t} R\left(t-t_{k}\right) I_{k}\left(\vartheta\left(t_{k}^{-}\right)\right)+\int_{0}^{t} R(t-u) B \Gamma^{-1}\left\{\vartheta_{1}-R(b)[\varphi(0)-h(0, \varphi(-\delta(0)))]\right. \\
& -h(b, \vartheta(T-\delta(T)))-\int_{0}^{T} R(T-s) f(s, \vartheta(s-\rho(s))) d s-\sum_{0<t_{k}<T} R\left(T-t_{k}\right) I_{k}\left(\vartheta\left(t_{k}^{-}\right)\right) \\
& \left.-\int_{0}^{T} \int_{\mathcal{U}} R(T-s) q(s, \vartheta(s-\gamma(s)), z) \widehat{\eta}(d s, d z)-\int_{0}^{T} R(T-s) \sigma(s) d Z_{Q}^{H}(s)\right\}(u) d u .
\end{aligned}
$$

has a fixed point, which is then a mild solution for the stochastic impulsive integrodifferential system (1.1). Clearly, $\Psi \vartheta(T)=\vartheta_{1}$, which implies that the stochastic control $u$ steers the system from the initial state $\varphi$ to $\vartheta_{1}$ in time $b$, provided we can find a fixed point of the operator $\Psi$ which means that the system is controllable on $[-r, T]$. The proof is given in the following two steps. 
Step 1. $\Psi$ is well defined. Let $\vartheta \in \mathcal{B}_{\mathrm{T}}$ and $\mathrm{t} \in \mathrm{J}$, we are going to show that each function $\Psi(\vartheta)(\cdot)$ is continuous on $J$ in the $\mathcal{L}^{2}(\Omega, \mathbb{H})$-sense. Let $t \in(0, T)$ and $|\epsilon|$ be sufficiently small. Then for any fixed $\vartheta \in \mathcal{B}_{\mathrm{T}}$, we have

$$
\begin{aligned}
& \mathbf{E}\|(\Psi \vartheta)(\mathrm{t}+\epsilon)-(\Psi \vartheta)(\mathrm{t})\|_{\mathcal{B}_{\mathrm{T}}}^{2} \\
& \leqslant 7 \mathbf{E}\|[\mathrm{R}(\mathrm{t}+\epsilon)-\mathrm{R}(\mathrm{t})][\varphi(0)-\mathrm{h}(0, \varphi(-\delta(0)))]\|^{2}+7 \mathbf{E}\|\mathrm{h}(\mathrm{t}+\epsilon, \vartheta(\mathrm{t}+\epsilon-\delta(\mathrm{t}+\epsilon)))-\mathrm{h}(\mathrm{t}, \vartheta(\mathrm{t}-\delta(\mathrm{t})))\|^{2} \\
& +7 E\left\|\int_{0}^{t+\epsilon} R(t+\epsilon-s) f(s, \vartheta(s-\rho(s))) d s-\int_{0}^{t} R(t-s) f(s, \vartheta(s-\rho(s))) d s\right\|^{2} \\
& +7 \mathbf{E}\left\|\int_{0}^{t+\epsilon} \int_{\mathcal{U}} R(t+\epsilon-s) q(s, \vartheta(s-\gamma(s)), z) \widehat{\eta}(d s, d z)-\int_{0}^{t} \int_{\mathcal{U}} R(t-s) q(s, \vartheta(s-\gamma(s)), z) \widehat{\eta}(d s, d z)\right\|^{2} \\
& +7 \mathrm{E}\left\|\int_{0}^{\mathrm{t}+\epsilon} \mathrm{R}(\mathrm{t}+\epsilon-\mathrm{s}) \sigma(\mathrm{s}) \mathrm{d} \mathrm{Z}_{\mathrm{Q}}^{\mathrm{H}}(\mathrm{s})-\int_{0}^{\mathrm{t}} \mathrm{R}(\mathrm{t}-\mathrm{s}) \sigma(\mathrm{s}) \mathrm{d} \mathrm{Z}_{\mathrm{Q}}^{\mathrm{H}}(\mathrm{s})\right\|^{2} \\
& +7 \mathbf{E}\left\|\sum_{0<t_{k}<T}\left[R\left(t+\epsilon-t_{k}\right)-R\left(t-t_{k}\right)\right] I_{k}\left(\vartheta\left(t_{k}^{-}\right)\right)\right\|^{2} \\
& +7 \mathbf{E} \| \int_{0}^{t+\epsilon} \mathrm{R}(\mathrm{t}+\epsilon-\mathrm{u}) \mathbb{B} \Gamma^{-1}\left\{\vartheta_{1}-\mathrm{R}(\mathrm{T})[\varphi(0)-\mathrm{h}(0, \varphi(-\delta(0)))]\right. \\
& -h(T, \vartheta(T-\delta(T)))-\int_{0}^{T} R(T-s) f(s, \vartheta(s-\rho(s))) d s-\sum_{0<t_{k}<T} R\left(T-t_{k}\right) I_{k}\left(\vartheta\left(t_{k}^{-}\right)\right) \\
& \left.-\int_{0}^{T} \int_{U} R(T-s) q(s, \vartheta(s-\gamma(s)), z) \widehat{\eta}(d s, d z)-\int_{0}^{T} R(T-s) \sigma(s) d Z_{Q}^{H}(s)\right\}(u) d u \\
& -\int_{0}^{\mathrm{t}} \mathrm{R}(\mathrm{t}-\mathrm{u}) \mathrm{B} \Gamma^{-1}\left\{\vartheta_{1}-\mathrm{R}(\mathrm{T})[\varphi(0)-\mathrm{h}(0, \varphi(-\delta(0)))]\right. \\
& -h(T, \vartheta(T-\delta(T)))-\int_{0}^{T} R(T-s) f(s, \vartheta(s-\rho(s))) d s-\sum_{0<t_{k}<T} R\left(T-t_{k}\right) I_{k}\left(\vartheta\left(t_{k}^{-}\right)\right) \\
& \left.-\int_{0}^{T} \int_{\mathcal{U}} R(T-s) q(s, \vartheta(s-\gamma(s)), z) \widehat{\eta}(d s, d z)-\int_{0}^{T} R(T-s) \sigma(s) d Z_{Q}^{H}(s)\right\}(u) d u \|^{2} \\
& :=7 \sum_{i=1}^{7} \partial_{i}(\epsilon) \text {. }
\end{aligned}
$$

Strong's continuity of $R(t)$ permits us to deduce

$$
\lim _{\epsilon \rightarrow 0}[R(t+\epsilon)-R(t)][\varphi(0)-h(0, \varphi(-\delta(0)))]=0 .
$$

One can infer from Definition 2.3 (i) that

$$
\begin{aligned}
& \|[\mathrm{R}(\mathrm{t}+\epsilon)-\mathrm{R}(\mathrm{t})][\varphi(0)-\mathrm{h}(0, \varphi(-\delta(0)))]\|^{2} \\
& \quad \leqslant 2 \mathrm{M}^{2}\left[\mathrm{e}^{-2 \mu_{0}(\mathrm{t}+\epsilon)}+\mathrm{e}^{-2 \mu_{0} \mathrm{t}}\right]\|\varphi(0)-\mathrm{h}(0, \varphi(-\delta(0)))\|^{2} \in \mathcal{L}^{2}(\Omega) .
\end{aligned}
$$

As a result, the Lebesgue dominated theorem implies that

$$
\lim _{\epsilon \rightarrow 0} \mathcal{J}_{1}(\epsilon)=0
$$

On the basis of $\left(\mathbf{H}_{7}\right)$, we reach the conclusion that

$$
\lim _{\epsilon \rightarrow 0} \mathbf{E}\|\mathbf{h}(\mathrm{t}+\epsilon, \vartheta(t+\epsilon-\delta(t+\epsilon)))-\mathrm{h}(\mathrm{t}, \vartheta(t-\delta(t)))\|^{2}=0 .
$$


Thus

$$
\lim _{\epsilon \rightarrow 0} \mathcal{J}_{2}(\epsilon)=0 \text {. }
$$

Consider the case of $\mathcal{J}_{3}(\epsilon)$. Without loss of generality, we suppose that $\epsilon>0$ (the case $\epsilon<0$ is similar). We have

$$
\begin{aligned}
J_{3}(\epsilon) & \leqslant \mathbf{E}\left\|\int_{0}^{t+\epsilon} R(t+\epsilon-s) f(s, \vartheta(s-\rho(s))) d s-\int_{0}^{t} R(t-s) f(s, \vartheta(s-\rho(s))) d s\right\|^{2} \\
& \leqslant 2 E\left\|\int_{0}^{t}[R(t+\epsilon-s)-R(t-s)] f(s, \vartheta(s-\rho(s))) d s\right\|^{2}+2 E\left\|\int_{t}^{t+\epsilon} R(t+\epsilon-s) f(s, \vartheta(s-\rho(s))) d s\right\|^{2} \\
& :=2 J_{31}(\epsilon)+2 J_{32}(\epsilon) .
\end{aligned}
$$

When we apply the Hölder inequality on $\mathcal{J}_{31}(\epsilon)$, we obtain

$$
\partial_{31}(\epsilon) \leqslant \mathrm{tE} \int_{0}^{\mathrm{t}}\|[\mathrm{R}(\mathrm{t}+\epsilon-s)-\mathrm{R}(\mathrm{t}-\mathrm{s})] \mathrm{f}(\mathrm{s}, \vartheta(s-\rho(s)))\|^{2} \mathrm{ds} .
$$

Using Definition 2.3 (ii), for each $s \in[0, t]$, we have

$$
\lim _{\epsilon \rightarrow 0}[R(t+\epsilon-s)-R(t-s)] f(s, \vartheta(s-\rho(s)))=0 .
$$

In light of assumption $\left(\mathbf{H}_{5}\right)$ and Definition 2.3 (i), we can conclude that

$$
\begin{aligned}
& \|[R(t+\epsilon-s)-R(t-s)] f(s, \vartheta(s-\rho(s)))\|^{2} \\
& \quad \leqslant 2 M^{2}\left[e^{-2 \mu_{0}(t+\epsilon-s)}+e^{-2 \mu_{0}(t-s)}\right]\|f(s, \vartheta(s-\rho(s)))\|^{2} \in \mathcal{L}^{2}([0, t] \times \Omega) .
\end{aligned}
$$

In this case, too, the Lebesgue dominated convergence theorem implies

$$
\lim _{\epsilon \rightarrow 0} \partial_{31}(\epsilon)=0 .
$$

Furthermore, using the Hölder inequality and assumptions $\left(\mathbf{H}_{3}\right),\left(\mathbf{H}_{5}\right)$, we can derive

$$
\begin{aligned}
\partial_{32}(\epsilon) & \leqslant \int_{t}^{t+\epsilon}\|R(t+\epsilon-s)\|^{2} d s E \int_{t}^{t+\epsilon}\|f(s, \vartheta(s-\rho(s)))\|^{2} d s \\
& \leqslant \tilde{M}^{2}\left(2 \mu_{0}\right)^{-1} M_{f}\left[1-e^{-2 \mu_{0} \epsilon}\right] \int_{t}^{t+\epsilon}\left(1+E\|\vartheta(s-\rho(s))\|^{2}\right) d s \stackrel{\epsilon \rightarrow 0}{\longrightarrow} 0 .
\end{aligned}
$$

Thus, we obtain

$$
\lim _{\epsilon \rightarrow 0} \mathcal{J}_{3}(\epsilon)=0 .
$$

For the term $\mathcal{J}_{4}(\epsilon)$, we have by assumption $\left(\mathbf{H}_{3}\right)$ :

$$
\begin{aligned}
J_{4}(\epsilon) \leqslant & 2 E\left\|\int_{0}^{t} \int_{\mathcal{U}}[R(t+\epsilon-s)-R(t-s)] q(s, \vartheta(s-\gamma(s)), z) \widehat{\eta}(d s, d z)\right\|^{2} \\
& +2 E\left\|\int_{t}^{t+\epsilon} \int_{\mathcal{U}} R(t+\epsilon-s) q(s, \vartheta(s-\gamma(s)), z) \widehat{\eta}(d s, d z)\right\|^{2} \\
\leqslant & 2 \int_{0}^{t} \int_{\mathcal{U}} E\|[R(t+\epsilon-s)-R(t-s)] q(s, \vartheta(s-\gamma(s)), z)\|^{2} \lambda(d z) d s \\
& +2 \tilde{M}^{2} \int_{t}^{t+\epsilon} \int_{\mathcal{U}} e^{-\mu_{0}(t+\epsilon-s)}\left(\|q(s, \vartheta(s-\gamma(s)), z)\|^{2} \lambda(d z) d s\right. \\
:= & 2 \partial_{41}(\epsilon)+2 \mathcal{J}_{42}(\epsilon) .
\end{aligned}
$$


By $\left(\mathbf{H}_{3}\right)$, the strong continuity of $R(t)$ and Lebesgue dominated convergence theorem, we get $\lim _{\epsilon \rightarrow 0} \mathcal{J}_{41}(\epsilon)=$ 0 . Therefore

$$
\lim _{\epsilon \rightarrow 0} \mathcal{J}_{4}(\epsilon)=0
$$

For the term $\mathcal{J}_{5}(\epsilon)$, we have

$$
\begin{aligned}
J_{5}(\epsilon) & \leqslant 2 E\left\|\int_{0}^{t}[R(t+\epsilon-s)-R(t-s)] \sigma(s) d Z_{Q}^{H}(s)\right\|^{2}+2 E\left\|\int_{t}^{t+\epsilon} R(t+\epsilon-s) \sigma(s) d Z_{Q}^{H}(s)\right\|^{2} \\
& :=2 J_{51}(\epsilon)+2 J_{52}(\epsilon) .
\end{aligned}
$$

Lemma 2.2 implies that

$$
\partial_{51}(\epsilon) \leqslant C(H) T^{2 H-1} \int_{0}^{t}\|[R(t+\epsilon-s)-R(t-s)] \sigma(s)\|_{\mathcal{L}_{2}^{0}}^{2} d s .
$$

Based on strong continuity of $R(t)$, for each $s \in[0, t]$ the following limit holds:

$$
\lim _{\epsilon \rightarrow 0}\|[R(t+\epsilon-s)-R(t-s)] \sigma(s)\|_{\mathcal{L}_{2}^{0}}^{2}=0
$$

By $\left(\mathbf{H}_{3}\right)$ and Lebesgue dominated theorem, we have

$$
\|[R(t+\epsilon-s)-R(t-s)] \sigma(s)\|^{2} \leqslant 2 M^{2}\left[e^{-2 \mu_{0}(t+\epsilon-s)}+e^{-2 \mu_{0}(t-s)}\right]\|\sigma(s)\|_{\mathcal{L}_{2}^{0}}^{2} \in \mathcal{L}^{1}(J, d s)
$$

and

$$
\lim _{\epsilon \rightarrow 0} \mathcal{J}_{51}(\epsilon)=0
$$

Applying Lemma 2.2 to $\mathcal{J}_{52}(\epsilon)$ we obtain

$$
J_{52}(\epsilon) \leqslant 2 \mathrm{HM}^{2} \epsilon^{2 \mathrm{H}-1} \int_{\mathrm{t}}^{\mathrm{t}+\epsilon} e^{-2 \mu_{0} s}\|\sigma(s)\|_{\mathcal{L}_{2}^{0}}^{2} \mathrm{~d} s \stackrel{\epsilon \rightarrow 0}{\longrightarrow} 0 .
$$

Thus,

$$
\lim _{\epsilon \rightarrow 0} \partial_{52}(\epsilon)=0
$$

Therefore

$$
\lim _{\epsilon \rightarrow 0} \partial_{5}(\epsilon)=0
$$

Now, we have

$$
\partial_{6}(\epsilon) \leqslant \sum_{0<t_{k}<T}\left[\left\|R\left(t+\epsilon-t_{k}\right)-R\left(t-t_{k}\right)\right\|^{2}\right] E\left\|I_{k}\left(\vartheta\left(t_{k}^{-}\right)\right)\right\|^{2} .
$$

By the assumptions $\left(\mathbf{H}_{1}\right),\left(\mathbf{H}_{6}\right)$, and the strong continuity of $R(t)$, one has that

$$
\lim _{\epsilon \rightarrow 0} \mathfrak{J}_{6}(\epsilon)=0
$$

For the estimation of term $\mathcal{J}_{7}(\epsilon)$, we have

$$
\begin{aligned}
\partial_{7}(\epsilon) \leqslant & 2 E \| \int_{0}^{t}[R(t+\epsilon-u)-R(t-u)] B \Gamma^{-1}\left\{\vartheta_{1}-R(T)[\varphi(0)-h(0, \varphi(-\delta(0)))]\right. \\
& -h(T, \vartheta(T-\delta(T)))-\int_{0}^{T} R(T-s) f(s, \vartheta(s-\rho(s))) d s-\sum_{0<t_{k}<T} R\left(T-t_{k}\right) I_{k}\left(\vartheta\left(t_{k}^{-}\right)\right)
\end{aligned}
$$




$$
\begin{aligned}
& \left.-\int_{0}^{T} \int_{\mathcal{U}} R(T-s) q(s, \vartheta(s-\gamma(s)), z) \widehat{\eta}(d s, d z)-\int_{0}^{T} R(T-s) \sigma(s) d Z_{Q}^{H}(s)\right\}(u) d u \|^{2} \\
& +2 E \| \int_{t}^{t+\epsilon} R(t+\epsilon-u) B \Gamma^{-1}\left\{\vartheta_{1}-R(T)[\varphi(0)-h(0, \varphi(-\delta(0)))]\right. \\
& -h(T, \vartheta(T-\delta(T)))-\int_{0}^{T} R(T-s) f(s, \vartheta(s-\rho(s))) d s-\sum_{0<t_{k}<T} R\left(T-t_{k}\right) I_{k}\left(\vartheta\left(t_{k}^{-}\right)\right) \\
& \left.-\int_{0}^{T} \int_{U} R(T-s) q(s, \vartheta(s-\gamma(s)), z) \widehat{\eta}(d s, d z)-\int_{0}^{T} R(T-s) \sigma(s) d Z_{Q}^{H}(s)\right\}(u) d u \|^{2} \\
& :=J_{71}(\epsilon)+J_{72}(\epsilon) .
\end{aligned}
$$

Since

$$
\begin{aligned}
\lim _{\epsilon \rightarrow 0} \| & {[R(t+\epsilon-u)-R(t-u)] B \Gamma^{-1}\left\{\vartheta_{1}-R(T)[\varphi(0)-h(0, \varphi(-\delta(0)))]\right.} \\
& -h(T, \vartheta(T-\delta(T)))-\int_{0}^{T} R(T-s) f(s, \vartheta(s-\rho(s))) d s-\sum_{0<t_{k}<T} R\left(T-t_{k}\right) I_{k}\left(\vartheta\left(t_{k}^{-}\right)\right) \\
& \left.-\int_{0}^{T} \int_{\mathcal{U}} R(T-s) q(s, \vartheta(s-\gamma(s)), z) \widehat{\eta}(d s, d z)-\int_{0}^{T} R(b-s) \sigma(s) d Z_{Q}^{H}(s)\right\} \|^{2}=0
\end{aligned}
$$

and

$$
\begin{aligned}
\|[ & R(t+\epsilon-u)-R(t-u)] B \Gamma^{-1}\left\{\vartheta_{1}-R(T)[\varphi(0)-h(0, \varphi(-\delta(0)))]\right. \\
& -h(T, \vartheta(T-\delta(T)))-\int_{0}^{T} R(T-s) f(s, \vartheta(s-\rho(s))) d s-\sum_{0<t_{k}<T} R\left(T-t_{k}\right) I_{k}\left(\vartheta\left(t_{k}^{-}\right)\right) \\
& \left.-\int_{0}^{T} \int_{\mathcal{U}} R(T-s) q(s, \vartheta(s-\gamma(s)), z) \widehat{\eta}(d s, d z)-\int_{0}^{T} R(T-s) \sigma(s) d Z_{Q}^{H}(s)\right\}(u) \|^{2} \\
\leqslant & 14 M^{2}\left(e^{-2 \mu_{0}(t+\epsilon-u)}+e^{-2 \mu_{0}(t-u)}\right) M_{\mathbb{B}} M_{\Gamma}\left\{E\left\|\vartheta_{1}\right\|^{2}+\tilde{M}^{2} e^{-2 \mu_{0} T} E\|\varphi(0)-h(0, \varphi(-\delta(0)))\|^{2}\right. \\
& +M_{h}\left(1+\sup _{s \in[-r, T]} E\|\vartheta(s)\|^{2}\right)+T \tilde{M}^{2}\left(2 \mu_{0}\right)^{-1} M_{f}\left(1+\sup _{s \in[-r, T]} E\|\vartheta(s)\|^{2}\right) \\
& +\tilde{M}^{2}\left(\sum_{k=1}^{\infty} d_{k}\right)^{2}\left(1+\sup _{s \in[-r, T]} E\|\vartheta(s)\|^{2}\right)+T \tilde{M}^{2} M_{q}^{2}\left(2 \mu_{0}\right)^{-1}\left(1+\sup _{s \in[-r, T]} E\|\vartheta(s)\|^{2}\right) \\
& \left.+\tilde{M}^{2}\left(2 \mu_{0}\right)^{-1} c(H) T^{2 H}-1 \int_{0}^{T}\|\sigma(s)\|_{\mathcal{L}_{2}^{0}}^{2} d s\right\} \in \mathcal{L}^{1},
\end{aligned}
$$

we conclude, by the dominated convergence theorem that

$$
\lim _{\epsilon \rightarrow 0} \mathcal{J}_{71}(\epsilon)=0 .
$$


From the assumptions $\left(\mathbf{H}_{3}\right)-\left(\mathbf{H}_{9}\right)$ and applying Lemma 2.2 to $\mathcal{J}_{71}(\epsilon)$ we obtain

$$
\begin{aligned}
& \partial_{72}(\epsilon) \leqslant \tilde{M}^{2}\left(2 \mu_{0}\right)^{-1}\left[1-e^{-2 \mu_{0} \epsilon}\right] M_{\mathbb{B}} M_{\Gamma} \int_{t}^{t+\epsilon}\left\{\mathbf{E}\left\|\vartheta_{1}\right\|^{2}\right. \\
& +\tilde{M}^{2} e^{-2 \mu_{0} \mathrm{~T}} \mathbf{E}\|\varphi(0)-\mathrm{h}(0, \varphi(-\delta(0)))\|^{2}+\mathrm{M}_{\mathrm{h}}\left(1+\sup _{\mathrm{s} \in[-\mathrm{r}, \mathrm{T}]} \mathbf{E}\|\vartheta(\mathrm{s})\|^{2}\right) \\
& +b \tilde{M}^{2}\left(2 \mu_{0}\right)^{-1} M_{f}\left(1+\sup _{s \in[-r, T]} E\|\vartheta(s)\|^{2}\right)+\tilde{M}^{2}\left(\sum_{k=1}^{\infty} d_{k}\right)^{2}\left(1+\sup _{s \in[-r, T]} \mathbf{E}\|\vartheta(s)\|^{2}\right) \\
& +\mathrm{T} \tilde{M}^{2} M_{\mathrm{q}}^{2}\left(2 \mu_{0}\right)^{-1}\left(1+\sup _{s \in[-r, \mathrm{~T}]} \mathbf{E}\|\vartheta(s)\|^{2}\right) \\
& \left.+\tilde{M}^{2}\left(2 \mu_{0}\right)^{-1} c(H) T^{2 H-1} \int_{0}^{b}\|\sigma(s)\|_{\mathcal{L}_{2}^{0}}^{2} \mathrm{ds}\right\} \mathrm{d} u \rightarrow 0 \text { as } \epsilon \rightarrow 0 \text {. }
\end{aligned}
$$

Replacing (3.4)-(3.15) in (3.3), we deduce

$$
\lim _{\epsilon \rightarrow 0} \mathbf{E}\|(\Psi \vartheta)(t+\epsilon)-(\Psi \vartheta)(t)\|^{2}=0
$$

Thus, the function $t \rightarrow(\Psi \vartheta)(t)$ is continuous on $J$.

Step 2. In this part of the proof, we will prove that $\Psi$ is a contraction mapping in $\mathcal{B}_{\mathrm{T}}$ with some $\mathrm{T}_{1}<\mathrm{T}$ to be specified later. Let $\vartheta, \chi \in \mathcal{B}_{\mathrm{T}_{1}}$ and $t \in[0, \mathrm{~T}]$. We have

$\mathbf{E}\|(\Psi \vartheta)(t)-(\Psi \chi)(t)\|^{2}$

$$
\begin{aligned}
\leqslant & 5 E\|h(t, \vartheta(t-\delta(t)))-h(t, \chi(t-\delta(t)))\|^{2}+5 E\left\|\int_{0}^{t} R(t-s)[f(s, \vartheta(s-\rho(s)))-f(s, \chi(s-\rho(s)))] d s\right\|^{2} \\
& +5 E\left\|\int_{0}^{t} \int_{\mathcal{U}} R(t-s)[q(s, \vartheta(s-\gamma(s)), z)-q(s, \chi(s-\gamma(s)), z)] \widehat{\eta}(d s, d z)\right\|^{2} \\
& +5 E\left\|\sum_{0<t_{k}<T} R\left(t-t_{k}\right)\left[I_{k}\left(\vartheta\left(t_{k}^{-}\right)\right)-I_{k}\left(\chi\left(t_{k}^{-}\right)\right)\right]\right\|^{2} \\
& +5 E \| \int_{0}^{t} R(t-u) B \Gamma^{-1}\{-[h(T, \vartheta(T-\delta(T)))-h(T, \chi(T-\delta(T)))] \\
& -\int_{0}^{T} R(T-s)[f(s, \vartheta(s-\rho(s)))-f(s, \chi(s-\rho(s)))] d s-\sum_{0<t_{k}<T} R\left(T-t_{k}\right)\left[I_{k}\left(\vartheta\left(t_{k}^{-}\right)\right)-I_{k}\left(\chi\left(t_{k}^{-}\right)\right)\right] \\
& \left.-\int_{0}^{T} \int_{u} R(T-s)[q(s, \vartheta(s-\gamma(s)), z)-q(s, \chi(s-\gamma(s)), z)] \widehat{\eta}(d s, d z)\right\}(u) d u \|^{2} .
\end{aligned}
$$

Using (3.2), we have

$$
\begin{aligned}
& \mathbf{E}\|(\Psi \vartheta)(t)-(\Psi \chi)(t)\|^{2} \\
& \leqslant 5 E\|h(t, \vartheta(t-\delta(t)))-h(t, \chi(t-\delta(t)))\|^{2}+5 E\left\|\int_{0}^{t} R(t-s)[f(s, \vartheta(s-\rho(s)))-f(s, \chi(s-\rho(s)))] d s\right\|^{2} \\
& \quad+5 E\left\|\int_{0}^{t} \int_{\mathcal{U}} R(t-s)[q(s, \vartheta(s-\gamma(s)), z)-q(s, \chi(s-\gamma(s)), z)] \widehat{\eta}(d s, d z)\right\|^{2} \\
& \quad+5 E\left\|\sum_{0<t_{k}<T} R\left(t-t_{k}\right)\left[I_{k}\left(\vartheta\left(t_{k}^{-}\right)\right)-I_{k}\left(\chi\left(t_{k}^{-}\right)\right)\right]\right\|^{2}
\end{aligned}
$$




$$
\begin{aligned}
& +5 \mathbf{E} \| \int_{0}^{t} R(t-u) B \Gamma^{-1}\{-[h(T, \vartheta(T-\delta(T)))-h(T, \chi(T-\delta(T)))] \\
& -\int_{0}^{T} R(T-s)[f(s, \vartheta(s-\rho(s)))-f(s, \chi(s-\rho(s)))] d s-\sum_{0<t_{k}<T} R\left(T-t_{k}\right)\left[I_{k}\left(\vartheta\left(t_{k}^{-}\right)\right)-I_{k}\left(\chi\left(t_{k}^{-}\right)\right)\right] \\
& \left.-\int_{0}^{T} \int_{\mathcal{U}} R(b-s)[q(s, \vartheta(s-\gamma(s)), z)-q(s, \chi(s-\gamma(s)), z)] \widehat{\eta}(d s, d z)\right\}(u) d u \|^{2} .
\end{aligned}
$$

By using Definition 2.3, Hölder inequality and assumptions $\left(\mathbf{H}_{3}\right)-\left(\mathbf{H}_{7}\right),\left(\mathbf{H}_{9}\right)$, we get

$$
\begin{aligned}
\mathbf{E}\|(\Psi \vartheta)(t)-(\Psi \chi)(t)\|^{2} & \\
\leqslant & 5 C_{\mathrm{h}}^{2} \sup _{s \in[-r, t]} E\|\vartheta(s-\delta(s))-\chi(s-\delta(s))\|^{2} \\
& +5 \tilde{M}^{2}\left(2 \mu_{0}\right)^{-1} t C_{f}^{2} \sup _{s \in[-r, t]} E\|\vartheta(s-\rho(s))-\chi(s-\rho(s))\|^{2}+5 \tilde{M}^{2}\left(\sum_{k=1}^{\infty} C_{I_{k}}\right)^{2} \sup _{s \in[-r, t]} E\|\vartheta(s)-\chi(s)\|^{2} \\
& +5 \tilde{M}^{2}\left(2 \mu_{0}\right)^{-1} t C_{\mathrm{q}}^{2} \sup _{s \in[-r, t]} E\|\vartheta(s-\gamma(s))-\chi(s-\gamma(s))\|^{2} \\
& +20\left(2 \mu_{0}\right)^{-1} \tilde{M}^{2}\left(1-e^{-\mu_{0} t}\right) M_{\mathbb{B}} M_{\Gamma}\left\{C_{h}^{2} \sup _{s \in[-r, T]} E\|\vartheta(s-\delta(s))-\chi(s-\delta(s))\|^{2}\right. \\
& +\tilde{M}^{2}\left(2 \mu_{0}\right)^{-1} \mathrm{TC}_{\mathrm{f}}^{2} \sup _{s \in[-r, T]} E\|\vartheta(s-\rho(s))-\chi(s-\rho(s))\|^{2}+\tilde{M}^{2}\left(\sum_{k=1}^{\infty} C_{I_{k}}\right)^{2} \sup _{s \in[-r, T]} E\|\vartheta(s)-\chi(s)\|^{2} \\
& \left.+\tilde{M}^{2}\left(2 \mu_{0}\right)^{-1} \mathrm{TC}_{\mathrm{q}}^{2} \sup _{s \in[-r, T]} E\|\vartheta(s-\gamma(s))-\chi(s-\gamma(s))\|^{2}\right\} .
\end{aligned}
$$

Hence, we have

$$
\sup _{s \in[-r, t]} \mathbf{E}\|(\Psi \vartheta)(s)-(\Psi \chi)(s)\|^{2} \leqslant \alpha(t) \sup _{s \in[-r, t]} \mathbf{E}\|(\Psi \vartheta)(s)-(\Psi \chi)(s)\|^{2}
$$

where

$$
\begin{aligned}
\alpha(t) \leqslant & 5 C_{h}^{2}+5 \tilde{M}^{2}\left(2 \mu_{0}\right)^{-1} t C_{f}^{2}+5 \tilde{M}^{2}\left(\sum_{k=1}^{\infty} C_{I_{k}}\right)^{2}+5 \tilde{M}^{2}\left(2 \mu_{0}\right)^{-1} t C_{q}^{2} \\
& +20\left(2 \mu_{0}\right)^{-1} \tilde{M}^{2}\left(1-e^{-\mu_{0} t}\right) M_{B} M_{\Gamma}\left\{C_{h}^{2}+\tilde{M}^{2}\left(2 \mu_{0}\right)^{-1} T C_{f}^{2}+\tilde{M}^{2}\left(\sum_{k=1}^{\infty} C_{I_{k}}\right)^{2}+\tilde{M}^{2}\left(2 \mu_{0}\right)^{-1} b C_{q}^{2}\right\} .
\end{aligned}
$$

By inequality (3.1), we have

$$
\alpha(0)=5 \mathrm{C}_{\mathrm{h}}^{2}+5 \tilde{\mathrm{M}}^{2}\left(\sum_{\mathrm{k}=1}^{\infty} \mathrm{C}_{\mathrm{I}_{\mathrm{k}}}\right)^{2}<1 .
$$

Then there exists $0<\mathrm{T}_{1} \leqslant \mathrm{~T}$ such that $0<\alpha(0)<1$ and the operator $\Psi$ is a contraction on $\mathcal{B}_{\mathrm{T}_{1}}$ and hence it has a unique fixed point on $\left[-r, T_{1}\right]$, which is a mild solution of system (1.1) on the interval $\left[-r, T_{1}\right]$. By repeating a similar process the solution can be extended to the entire interval $[-r, b]$. Therefore, the system (1.1) is controllable on $[-r, T]$.

The proof is complete. 


\section{Example}

We consider the following nonlocal stochastic integrodifferential system to illustrate the previous theoretical results.

$$
\left\{\begin{array}{l}
d\left[w(t, \xi)-\beta_{1} w(t-\rho(t), \xi)\right]=\left[\frac{\partial^{2}}{\xi^{2}}\left[w(t, \xi)-\beta_{1} w(t-\rho(t), \xi)\right]\right. \\
\left.+\int_{0}^{t} g(t-s) \frac{\partial^{2}}{\xi^{2}}\left[w(s, \xi)-\beta_{1} w(s-\rho(s), \xi)\right] d s+\beta_{2} w(t-\delta(t), \xi)+k(\xi) u(t)\right] d t \\
+\int_{u} \beta_{3} y(w(t-r(t), \xi)) \tilde{N}(d t, d y)+e^{-t} d Z_{Q}^{H}(t), \quad 0 \leqslant \xi \leqslant \pi, \quad t \neq t_{k}, \quad t \in[0, T] \\
\Delta w\left(t_{k}, \cdot\right)(\xi)=\frac{\beta_{4}}{2^{k}} w\left(t_{k}^{-}, \xi\right), \quad t=t_{k}, k=1,2, \ldots, \\
\vartheta(\theta, \cdot)=\vartheta_{0}(\theta, \xi) \in \mathbb{X}=L^{2}([0, \pi]), \quad \vartheta_{0}(\cdot, \xi) \in \mathcal{C}([-r, 0], \mathbb{R}), \quad \theta \in[0, \pi]
\end{array}\right.
$$

where $H \in\left(\frac{1}{2}, 1\right), Z_{Q}^{H}$ denotes standard Rosenblatt process defined on a stochastic basis $(\Omega, \mathcal{F}, \mathbb{P}), \beta_{1}, \beta_{2}$, $\beta_{3}, \beta_{4}$ are positive constants, $\mathcal{U}=\{v \in \mathbb{R}: 0<|v| \leqslant c, c>0\}, \mathrm{g}: \mathbb{R}^{+} \rightarrow \mathbb{R}^{+}$is continuous functions and $\varphi(\theta, \cdot) \in \mathrm{L}^{2}[0, \pi]$ is measurable and satisfies $\mathbb{E}\|\varphi\|^{2}<\infty$. Let $\mathbb{X}=\mathbb{Y}=\mathbb{U}=\mathrm{L}^{2}([0, \pi])$ with the norm $\|\cdot\|$. Define $\mathrm{A}: \mathrm{D}(\mathrm{A}) \subset \mathbb{X} \rightarrow \mathbb{X}$ by $\mathrm{A} x=x^{\prime \prime}$ with domain

$$
\mathrm{D}(\mathrm{A})=\left\{x(.) \in \mathbb{X}, x, x^{\prime} \text { are absolutely continuous, } x^{\prime \prime} \in \mathbb{X}, x(0)=x(\pi)\right\} .
$$

The spectrum of $A$ consists of the eigenvalues $-n^{2}$ for $n \in \mathbb{N}$, with associated eigenvectors

$$
e_{n}:=\sqrt{\frac{2}{\pi}} \sin (n x), \quad(n=1,2,3, \ldots) .
$$

Furthermore, the set $\left\{e_{n}: n \in \mathbb{N}\right\}$ is an orthogonal basis in $\mathbb{X}$. Then

$$
\mathrm{A} x=\sum_{n=1}^{\infty} \mathrm{n}^{2}\left\langle x, e_{\mathrm{n}}\right\rangle e_{\mathrm{n}}, \quad x \in \mathbb{X} .
$$

It is well known that $\mathrm{A}$ is the infinitesimal generator of a strongly continuous semigroup $\{\mathbf{T}(\mathbf{t})\}_{\mathbf{t} \geqslant 0}$ on $\mathbb{X}$, which is compact and is given by

$$
\mathrm{T}(\mathrm{t}) \mathrm{x}=\sum_{\mathrm{n}=1}^{\infty} \mathrm{e}^{-\mathrm{n}^{2} \mathrm{t}}<x, \mathrm{e}_{\mathrm{n}}>\mathrm{e}_{\mathrm{n}}, \quad x \in \mathbb{X} .
$$

Let $\Upsilon: \mathrm{D}(\mathrm{A}) \subset \mathbb{X} \rightarrow \mathbb{X}$ be the operator defined by

$$
\Upsilon(t)(\tilde{z})=g(t) A \tilde{z} \text { for } t \geqslant 0 \text { and } \tilde{z} \in D(A) .
$$

Further, define $\mathbb{B} \in \mathrm{L}(\mathrm{R}, \mathbb{H})$ by $\mathbb{B u}(\mathrm{t})=\mathrm{k}(\xi) \mathrm{u}, 0 \leqslant \xi \leqslant \pi, \mathrm{u} \in \mathbb{R}, \mathrm{k}(\xi) \in \mathrm{L}^{2}(0, \pi)$. Let $\Gamma \mathrm{u}=\int_{0}^{\mathrm{T}} \mathcal{R}(\mathrm{T}-$ s) $\mathbb{B u}(s) d s$, then we claim that $\Gamma$ is bounded due to Hölder inequality. Take $\operatorname{Ker}(\Gamma)=\left\{u \in \mathrm{L}^{2}([0, T], \mathbb{R})\right.$ : $\Gamma \mathrm{u}=0\}$ as a null space of $\Gamma$ and let $(\operatorname{Ker}(\Gamma))^{\perp}$ be its complement in $\mathrm{L}^{2}([0, \mathrm{~T}], \mathbb{R})$. Also take $\Gamma_{0}:(\operatorname{Ker}(\Gamma))^{\perp} \rightarrow$ Range $(\Gamma)$ is the restriction of $\Gamma$ to $(\operatorname{Ker}(\Gamma))^{\perp} . \Gamma_{0}$ is one-to-one operator. By using the inverse mapping theorem, we get $\Gamma^{-1}$ is bounded. Now, $\Gamma^{-1}$ is bounded and have 0 values in $L^{2}(J, U) \backslash \operatorname{Ker}(W)$, that is, $\left(\mathbf{H}_{9}\right)$ is satisfied. by

Define the operators $\mathrm{h}, \mathrm{f}:[0, \infty) \times \mathbb{X} \longrightarrow \mathbb{X}, \mathrm{q}:[0, \infty) \times \mathbb{H} \mapsto \mathbb{H}, \mathrm{q}:[0, \infty) \times \mathbb{H} \times \mathbb{H} \mapsto \mathbb{H}, \mathrm{I}_{\mathrm{k}}: \mathbb{H} \mapsto \mathbb{H}$

$$
\mathrm{h}(\mathrm{t}, w(\mathrm{t}-\rho(\mathrm{t})))(\xi)=\beta_{1} w(\mathrm{t}-\rho(\mathrm{t}), \xi), \xi \in[0, \pi],
$$




$$
\begin{aligned}
f(t, w(t-\rho(t)))(\xi) & =\beta_{2} w(t-\rho(t), \xi), \xi \in[0, \pi] \\
\mathrm{q}(\mathrm{t}, \phi, y)(\xi) & =\beta_{3} y w(t-r(t), \xi), \xi \in[0, \pi] \\
\mathrm{I}_{k}\left(w\left(\mathrm{t}_{\mathrm{k}}, \cdot\right)(\xi)\right. & =\frac{\beta_{4}}{2^{\mathrm{k}}} w\left(\mathrm{t}_{\mathrm{k}}^{-}, \xi\right), \quad k=1,2, \ldots, \xi \in[0, \pi] .
\end{aligned}
$$

In order to rewrite system (4.1) in an abstract form in $\mathbb{X}$, we introduce the following notations

$$
\left\{\begin{array}{l}
\vartheta(t)=w(t, \xi) \text { for } t \geqslant 0 \text { and } \xi \in[0, \pi] \\
\varphi(t)(\tau)=w_{0}(t, \xi) \text { for } t \in[-r, 0] \text { and } \xi \in[0, \pi] .
\end{array}\right.
$$

Then equation (4.1) takes the following abstract form

$$
\left\{\begin{array}{l}
d[\vartheta(t)-h(t, \vartheta(t-\delta(t)))]=\left[A[\vartheta(t)-h(t, \vartheta(t-\delta(t)))]+\int_{0}^{t} \gamma(t-s)[\vartheta(s)-h(s, \vartheta(t-\delta(s)))] d s\right. \\
+f(t, \vartheta(t-\rho(t)))+B u(t)] d t+\int_{u} q(t, \vartheta(t-\gamma(t)), z) \widehat{\eta}(d t, d z)+\sigma(t) d Z_{Q}^{H}(t), \quad t \neq t_{k} \in J:=[0, T], \\
\Delta\left(t_{k}\right)=\vartheta\left(t_{k}^{+}\right)-\vartheta\left(t_{k}^{-}\right)=I_{k}\left(t_{k}^{-}\right), \quad k=1,2, \ldots, \\
\vartheta_{0}(t)=\varphi(\cdot) \in \mathcal{C}_{\mathcal{F}_{0}}^{0}\left([-r, 0], \mathcal{L}^{2}(\Omega, \mathbb{H})\right), \quad t \in[-r, 0] .
\end{array}\right.
$$

Moreover, if $\mathrm{g}$ is bounded and $\mathrm{C}^{1}$ function such that $\mathrm{g}^{\prime}$ is bounded and uniformly continuous, then $\left(\mathbf{H}_{1}\right)$ and $\left(\mathbf{H}_{2}\right)$ are satisfied, and hence, by Theorem 2.4, Eq. (2.4) has a resolvent operator $(R(t))_{t \geqslant 0}$ on $\mathbb{X}$. Using [14, Lemma 5.2], let $\mu>\delta>1$ and $g(t)<\exp (-\beta t)$, for all $t \geqslant 0$. Then the above resolvent operator decays exponentially to zero. Specifically, $\|R(t)\| \leqslant \exp (-a t)$ where $a=1-1 / \delta$. It is obvious that all the assumptions are satisfied with

$$
\begin{aligned}
& \mu_{0}=1-\frac{1}{\delta}, \tilde{M}=1, C_{h}=\beta_{1} M_{h}=\beta_{1}^{2}, C_{f}=\beta_{2} M_{f}=\beta_{2}^{2}, \\
& C_{q}=M_{q}=\int_{\mathcal{U}} \beta_{3}^{2} y^{2} \lambda(d y), C_{I_{k}}=\frac{\beta_{4}}{2^{k}}, \quad d_{k}=\frac{\beta_{4}}{2^{2 k}} .
\end{aligned}
$$

Thus, by Theorem 3.1, Equation (4.1) is controllable on $J=[0, T]$ provided

$$
\beta_{1}^{2}+\left(\sum_{k=1}^{+\infty} C_{I_{k}}\right)^{2}<\frac{1}{5} .
$$

\section{Conclusion}

The controllability of impulsive neutral stochastic delay integrodifferential equations driven by Rosenblatt process and Lévy noise in Hilbert spaces is investigated in this paper. A novel set of adequate criteria is generated by employing a fixed point technique without imposing a strict compactness condition on the resolvent operator. The findings in this paper constitute a generalization and extension of the recent findings on this topic. An example is offered to demonstrate the theoretical conclusion reached. Furthermore, this result could be extended to investigate non-instantaneous impulsive neutral stochastic delay integrodifferential equations driven by Rosenblatt process and Lévy noise in Hilbert space.

\section{Acknowledgements}

The authors would like to thank the referees and the editor for their careful comments and valuable suggestions on this work. 


\section{References}

[1] P. Abry, V. Pipiras, Wavelet-based synthesis of the Rosenblatt process, Signal Process, 86 (2006), 2326-2339. 2.1

[2] H. M. Ahmed, Approximate controllability of impulsive neutral stochastic differential equations with fractional Brownian motion in a Hilbert space, Adv. Differential Equ., 2014 (2014), 11 pages. 1

[3] C. T. Anh, L. V. Hien, Exponential stability of solutions to semilinear parabolic equations with delays, Taiwanese J. Math., 16 (2012), 2133-2151.

[4] G. Arthi, K. Balachandran, Controllability of damped second-order neutral functional differential systems with impulses, Taiwanese J. Math., 16 (2012), 89-106. 1

[5] A. Boudaoui, E. Lakhel, Controllability of stochastic impulsive neutral functional differential equations driven by fractional Brownian motion with infinite delay, Differ. Equ. Dyn. Syst., 26 (2018), 247-263. 1

[6] A. Boudaoui, E. Lakhel, Controllability of impulsive neutral stochastic integrodifferentialsystems driven by fractional Brownian motion with delay and Poisson jumps, Proyecciones (Antofagasta, Online), 2021 (2021), 18 pages.

[7] F. E. Browder, W. V. Petryshyn, Construction of fixed points of nonlinear mappings in Hilbert spaces, J. Math. Anal. Appl., 20 (1967), 197-228. 1

[8] M. Chen, Approximate controllability of stochastic equations in a Hilbert space with fractional Brownian motions, Stoch. Dyn., 15 (2015), 16 pages. 1

[9] E. N. Chukwu, Differential Models and Neutral Systems for Controlling the Wealth of Nations, World Scientific Publishing Co., River Edge, (2001). 1

[10] J. Cui, L. T. Yan, Controllability of neutral stochastic evolution equations driven by fractional Brownian motion, Acta Math. Sci. Ser. B (Engl. Ed.), 37 (2017), 108-118. 1

[11] G. Da Prato, J. Zabczyk, Stochastic Equations in Infinite Dimensions, Cambridge University Press, Cambridge, (2014). 1

[12] B. de Andrade, J. P. C. Dos Santos, Existence of solutions for a fractional neutral integro-differential equation with unbounded delay, Electron. J. Differential Equations, 2012 (2012), 13 pages. 1

[13] A. Debbouche, J. J. Nieto, Sobolev type fractional abstract evolution equations with nonlocal conditions and optimal multi-controls, Appl. Math. Comput., 245 (2014), 74-85. 1

[14] M. Dieye, M. A. Diop, K. Ezzinbi, On exponential stability of mild solutions for some stochastics partial integrodifferential equations, Statist. Probab. Lett., 123 (2017), 61-76. 4

[15] R. L. Dobrushin, P. Major, Non-central limit theorems for non-linear functionals of Gaussian fields, Zeitschrift fur Wahrscheinlichkeitstheorie und Verwandte Gebiete, 50 (1979), 27-52. 2.1

[16] J. P. C. Dos Santos, H. Henriquez, E. Hernández, Existence results for neutral integro-differential equations with unbounded delay, J. Integral Equ. Appl., 23 (2011), 289-330. 1

[17] K. Ezzinbi, H. Toure, I. Zabsonre, Local existence and regularity of solutions for some partial functional integrodifferential equations with infinite delay in Banach space, Nonlinear Anal., 70 (2009), 3378-3389. 1

[18] L. Gawarecki, V. Mandrekar, Stochastic Differential Equations in Infinite Dimensions with Applications to Stochastic Partial Differential Equations, Probability and its Applications, Springer, Heidelberg, (2011).

[19] R. C. Grimmer, Resolvent operators for integral equations in a Banach space, Trans. Amer. Math. Soc., 273 (1982), 333-349. 2.2, 2.3, 2.2, 2.4

[20] J. K. Hale, K. R. Meyer, A class of functional equations of neutral type, merican Mathematical Society, Providence, (1967). 1

[21] J. K. Hale, S. M. Verduyn Lunel, Introduction to functional differential equations, Springer-Verlag, New York, (1993). 1

[22] H. R. Henriquez, J. P. C. Dos Santos, Differentiability of solutions of abstract neutral integro-differential equations, J. Integral Equations Appl., 25 (2013), 47-77. 1

[23] E. Hernandez, D. O'Regan, Controllability of Volterra Fredholm type systems in Banach spaces, J. Franklin Inst., 346 (2009), 95-101. 1

[24] D. D. Huan, On the controllability of nonlocal second-order impulsive neutral stochastic integro-differential equations with infinite delay, Asian J. Control, 17 (2015), 1233-1242. 1

[25] D. D. Huan, R. P. Agarwal, Asymptotic behavior, attracting and quasi-invariant sets for impulsive neutral SPFDE driven by Leoy noise, Stoch. Dyn., 18 (2018), 21 pages. 1

[26] D. D. Huan, R. P. Agarwal, Controllability for impulsive neutral stochastic delay partial differential equations driven by fBm and Léry noise, Stoch. Dyn., 21 (2021), 24 pages. 1

[27] D. D. Huan, H. J. Gao, Controllability of nonlocal second order impulsive neutral stochastic functional integro-differential equations with delay and Poisson jumps, Cogent Eng., 2 (2015), 1-16.

[28] K. Jeet, D. Bahuguna, Approximate controllability of nonlocal neutral fractional integro-differential equations with finite delay, J. Dyn. Control Syst., 22 (2016), 485-504. 1

[29] V. B. Kolmanovskii, A. Myshkis, Applied Theory of Functional Differential Equations, Kluwer, Dordrecht, (1992).

[30] V. B. Kolmanovskii, V. R. Nosov, Stability of neutral type functional differential equations, Nonlinear Anal., 6 (1982), 873-910. 1

[31] I. Kruk, F. Russo, C. A. Tudor, Wiener integrals, Malliavin calculus and covariance measure structure, J. Funct. Anal., 249 (2007), 92-142. 2.1 
[32] E. Lakhel, Neutral stochastic functional differential evolution equations driven by Rosenblatt process with varying-time delays, Proyecciones, 38 (2019), 665-689. 1

[33] E. Lakhel, S. Hajji, Neutral stochastic functional differential equations driven fractional Brownian motion and Poisson point processes, Gulf J. Math., 4 (2016), 1-14. 1

[34] N. N. Leonenko, V. V. Ahn, Rate of convergence to the Rosenblatt distribution for additive functionals of stochastic processes with long-range dependence, J. Appl. Math. Stochastic Anal., 14 (2001), 27-46. 2.1

[35] A. Lunardi, On the linear heat equation with fading memory, SIAM J. Math. Anal., 21 (1990), 1213-1224. 1

[36] M. Maejima, C. A. Tudor, Wiener integrals with respect to the Hermite process and a non central limit theorem, Stoch. Anal. Appl., 25 (2007), 1043-1056. 2.1, 2.1

[37] M. Maejima, C. A. Tudor, Selfsimilar processes with stationary increments in the second Wiener chaos, Probab. Math. Statist., 32 (2012), 167-186. 2.1

[38] M. Maejima, C. A. Tudor, On the distribution of the Rosenblatt process, Statist. Probab. Lett., 83 (2013), 1490-1495. 2.1

[39] N. I. Mahmudov, S. Zorlu, Approximate controllability of fractional integro-differential equations involving nonlocal initial conditions, Bound. Value Probl., 2013 (2013), 16 pages. 1

[40] N. I. Mahmudov, S. Zorlu, On the approximate controllability of fractional evolution equations with compact analytic semigroup, J. Comput. Appl. Math., 259 (2014), 194-204. 1

[41] K. Mathiyalagan, R. Sakthivel, J. H. Park, Robust reliable control for neutral-type nonlinear systems with time-varying delays, Rep. Math. Phys., 74 (2014), 181-203.

[42] X. R. Mao, Stochastic Differential Equations and Applications, Horwood Publishing Limited, Chichester, (1997). 1

[43] G. M. Mophou, Optimal control of fractional diffusion equation, Comput. Math. Appl., 61 (2011), 68-78. 1

[44] P. H. A. Ngoc, C. T. Tinh, New criteria for exponential stability of linear time-varying differential systems with delay, Taiwanese J. Math., 18 (2014), 1759-1774.

[45] B. Øksendal, Stochastic Differential Equations, 6th ed., Springer, New York, (2005). 1

[46] B. O'Neill, Semi-Riemannian geomerty with applications to relativity, Academic Press, London, (1983).

[47] J. Y. Park, K. Balachandtran, N. Annapoorani, Existence results for impulsive neutral functional integrodifferential equations with infinite delay, Nonlinear Anal., 71 (2009), 3152-3162. 1

[48] S. Peszat, J. Zabczyk, Stochastic Partial Differential Equations with Levy Noise, Cambridge University Press, Cambridge, (2007). 1

[49] V. Pipiras, M. S. Taqqu, Regularization and integral representations of Hermite processes, Statist. Probab. Lett., 80 (2010), 2014-2023. 2.1

[50] P. E. Protter, Stochastic Integration and Differential Equations, 2nd ed., Springer-Verlag, Berlin, (2004). 2.1

[51] M. D. Quinn, N. Carmichael, An approach to non-linear control problems using fixed point methods, degree theory and pseudo inverses, Numer. Func. Anal. Optim., 7 (1985), 197-219. 3

[52] R. Ravi Kumar, Nonlocal Cauchy problem for analytic resolvent integrodifferential equations in Banach spaces, Appl. Math. Comput., 204 (2008), 352-362. 1

[53] Y. Ren, H. L. Dai, R. Sakthivel, Approximate controllability of stochastic differential systems driven by a Leoy process, Internat. J. Control, 86 (2013), 1158-1164. 1

[54] R. Sakthivel, E. Anandhi, Approximate controllability of impulsive differential equations with state-dependent delay, Internat. J. Control, 83 (2010), 387-393. 1

[55] R. Sakthivel, N. I. Mahmudov, J. J. Nieto, Controllability for a class of fractional-order neutral evolution control systems, Appl. Math. Comput., 218 (2012), 10334-10340. 1

[56] R. Sakthivel, Y. Ren, Complete controllability of stochastic evolution equations with jumps, Rep. Math. Phys., 68 (2011), 163-174. 1

[57] M. S. Taqqu, Weak convergence to the fractional Brownian motion and to the Rosenblatt process, Zeitschrift fur Wahrscheinlichkeitstheorie und Verwandte Gebiete, 31 (1975), 287-302. 2.1

[58] S. Tindel, C. A. Tudor, F. Viens, Stochastic evolution equations with fractional Brownian motion, Probab. Theory Related Fields, 127 (2003), 186-204. 2.1

[59] R. Triggiani, A note on the lack of exact controllability for mild solutions in Banach spaces, SIAM J. Control Optim., 15 (1977), 407-411. 3

[60] C. A. Tudor, Analysis of the Rosenblatt process, ESAIM Probab. Stat., 12 (2008), 230-257. 2.1, 2.1, 2.1

[61] Y. H. Yao, Y. J. Cho, Y.-C. Liou, R. P. Agarwal, Constructed nets with perturbations for equilibrium and fixed point problems, J. Inequal. Appl., 2014 (2014), 14 pages. 